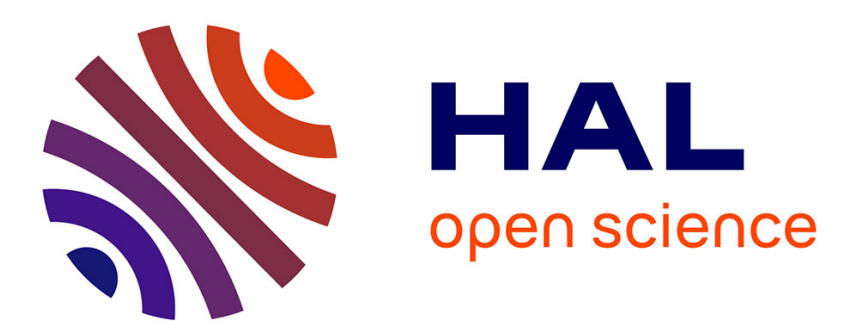

\title{
Pseudo-radar algorithms with two extremely wet months of disdrometer data in the Paris area
}

\author{
Auguste Gires, Ioulia Tchiguirinskaia, D Schertzer
}

\section{To cite this version:}

Auguste Gires, Ioulia Tchiguirinskaia, D Schertzer. Pseudo-radar algorithms with two extremely wet months of disdrometer data in the Paris area. Atmospheric Research, 2018, 203, pp.216-230. 10.1016/j.atmosres.2017.12.011 . hal-01704119

HAL Id: hal-01704119

https://hal-enpc.archives-ouvertes.fr/hal-01704119

Submitted on 8 Feb 2018

HAL is a multi-disciplinary open access archive for the deposit and dissemination of scientific research documents, whether they are published or not. The documents may come from teaching and research institutions in France or abroad, or from public or private research centers.
L'archive ouverte pluridisciplinaire HAL, est destinée au dépôt et à la diffusion de documents scientifiques de niveau recherche, publiés ou non, émanant des établissements d'enseignement et de recherche français ou étrangers, des laboratoires publics ou privés. 
Title: Pseudo-radar algorithms with two extremely wet months of disdrometer data in the Paris area

Authors:

Gires A., Tchiguirinskaia I., Schertzer D.

HMCo, Ecole des Ponts, UPE, Champs-sur-Marne, France

\begin{abstract}
:
Disdrometer data collected during the two extremely wet months of May and June 2016 at the Ecole des Ponts ParisTech are used to get insights on radar algorithms. The rain rate and pseudo-radar quantities (horizontal and vertical reflectivity, specific differential phase shift) are all estimated over several durations with the help of drop size distributions DSD) collected at $30 \mathrm{~s}$ time steps. The pseudo-radar quantities are defined with simplifying hypotheses, in particular on the DSD homogeneity. First it appears that the parameters of the standard radar relations $Z_{h}-R, R-K_{d p}$ and $R-Z_{h}-Z_{d r}$ for these pseudo-radar quantities exhibit strong variability between events and even within an event. Second an innovative methodology that relies on checking the ability of a given algorithm to reproduce the good scale invariant multifractal behaviour (on scales $30 \mathrm{~s}-\mathrm{few} \mathrm{h}$ ) observed on rainfall time series is implemented. In this framework, the classical hybrid model $\left(Z_{h}-R\right.$ for low rain rates and $R-K_{d p}$ for great ones) performs best, as well as the local estimates of the radar relations' parameters. However, we emphasise that due to the hypotheses on which they rely these observations cannot be straightforwardly extended to real radar quantities.
\end{abstract}

Key words: rainfall, disdrometer, radar algorithm, multifractal

\title{
1) Introduction
}

Disdrometers are rainfall point measurement devices that give access not only to rain rates but also to information about the size and velocity of drops falling through the sampling section. They are commonly implemented for research purposes and their operational use is spreading. Weather radars are basically the only devices providing volumetric rainfall data almost instantaneously, but in a less direct measurement. Indeed a radar transmits a wave and actually measures the power of the wave backscattered by the hydrometeors of the atmosphere, and not the rain rate which is the quantity hydrometeorologists are interested in. With the strong simplifying hypothesis that the drop size distribution (DSD) is homogeneous in the radar scanned volume and corresponds to the one measured with the help of disdrometer, it is possible to define pseudo radar quantities based on the pointwise disdrometer measurement. This furthermore assumes incoherent scattering, i.e. there is no clustering of the drops inside radar pulse volumes, whose radial scale is usually in the range of $100 \mathrm{~m}$ to $1 \mathrm{~km}$. In other words that drop centres are homogenously distributed. It has been shown that this hypothesis may lead to strong statistical biases (Lovejoy et al, 1996, Schertzer et al., 2012). These hypotheses enable to partially inverse the retrieval algorithms from radar backscattering to rain rate. This approach was implemented by numerous authors (Gires et al. 2016; Jaffrain and Berne 2012b; Leinonen et al. 2012; Ryzhkov et al. 2005; Verrier et al. 2013,). Others relied on synthetic DSD functions and ran various simulations using 
probability distributions for the key parameters characterizing the DSD (Anagnostou et al., 2010; Illingworth and Blackman, 2002; Ryzhkov et al., 2005).

In this paper we use data collected in May and June 2016 by the TARANIS observatory (exTreme and multi-scAle RAiNdrop parIS observatory, Gires et al. 2016) of the Fresnel Platform of Ecole des Ponts ParisTech (https://hmco.enpc.fr/Page/Fresnel-Platform/en). This period was extremely rainy over this area, and resulted in severe floods of the Seine River in Paris and its tributaries. The peak flow in Paris was reached on 7 June 2016 with levels not observed since 1982 on the Seine River. Some upstream tributaries of the Paris area, especially the Loing, reached levels greater than the ones of the famous 1910 flood (http://www.developpement-durable.gouv.fr/Point-sur-les-inondations-6-juin.html). This data is used to get insights on various radar algorithms.

The data is presented in section 2, with a highlight on two events exhibiting different features; an almost two day uninterrupted period of light rain (29-31 May 2016), and a short heavy one (17 June 2016). A multifractal analysis of the rainfall data is used to quantify the variability across scales of the measured time series (see Schertzer and Lovejoy 2011 for a recent review). The radar relations are investigated in section 3, after having defined the following pseudo-radar quantities: the horizontal reflectivity $Z_{\mathrm{h}}$, the specific phase shift $K_{\mathrm{dp}}$ and the differential reflectivity $Z_{\mathrm{dr}}$. The latter two depend on the oblate shape of drops. The parameters of the power law relations between these quantities and the rain rate are assessed on average over the whole period and also for each event. The variability during an event is studied as well. Finally in section 4, the consequences of the previous findings on rainfall retrieval with radars are analysed. More precisely reconstructed radar observations of rainfall time series (using the relations described in section 3) are compared with actual rainfall time series. An innovative comparison based on multifractal properties is carried out as well as the computation of standard scores commonly used by hydro-meteorologists.

\section{2) Rainfall data and preliminary analysis}

\section{1) Data collection and computation}

The data analysed in this paper was collected between 1 May 2016 and 31 June 2016 by the TARANIS observatory's optical disdrometers which are collocated on the roof of the Ecole des Ponts ParisTech building (East of Paris, France). Two OTT Parsivel ${ }^{2}$ disdrometers (Loffler-Mang and Joss 2000, for an initial version; Battaglia et al. 2010, OTT 2014) are the first type of disdrometer used and denoted Pars\#1 and Pars\#2 hereafter. The two Parsivel ${ }^{2}$ are installed perpendicularly. Such optical disdrometer transmits a laser sheet and computes the size and velocity of drops falling through the sampling area by assessing the portion of light occluded before it reaches the receiver. The second disdrometer type is a Campbell Scientific PWS100 (Ellis et al. 2006, Campbell Scientific Ltd 2012). In this case, it is the light refracted by drops that is analysed. It should be mentioned that a correction of the drops' diameters estimation was implemented to better take into account the oblateness of drops (Gires et al., 2016). Both devices basically provide a matrix with the number of drop(s) recorded according to classes of equivolumic diameter and terminal fall velocity. From this matrix, it is possible to compute the rain rate $R$ in $m m . h^{-1}$ (by counting the number of drop(s) that fell during a time interval) and a drop size distribution (DSD) denoted $N(D)$ (in $\mathrm{m}^{-3} \cdot \mathrm{mm}^{-1}$ ). With the aforementioned hypotheses (homogeneous DSD and distribution of the drop centres), the horizontal reflectivity $\left(Z_{\mathrm{h}}\right.$ in $\left.\mathrm{mm}^{6} \cdot \mathrm{m}^{-3}\right)$, the vertical reflectivity $\left(Z_{\mathrm{v}}\right.$ in $\left.\mathrm{mm}^{6} \cdot \mathrm{m}^{-3}\right)$ and the 
specific differential phase shift $\left(K_{\mathrm{dp}}\right.$ in $\left.{ }^{\circ} \cdot \mathrm{km}^{-1}\right)$ can be estimated as moments of the (local) DSD estimated by the disdrometers:

$$
\begin{aligned}
& Z_{h, v}=\frac{\lambda^{4}}{\pi^{5}\left|\frac{m^{2}-1}{m^{2}+1}\right|} \int_{D_{\min }}^{D_{\max }} N(D) \sigma_{B ; h, v}(D) d D \\
& K_{d p}=\frac{10^{-3} 1800 \lambda}{\pi} \int_{D_{\min }}^{D_{\max }} \operatorname{Re}\left[S_{h h}(D)-S_{v v}(D)\right] N(D) d D(2)
\end{aligned}
$$

where $D_{\min }$ and $D_{\max }$ are the minimum and maximum measured diameters $D$ 's (in mm), $\sigma_{B ; h, v}$ (in $\mathrm{mm}^{2}$ ) the backscattering cross section at horizontal / vertical polarization, $\operatorname{Re}\left(\mathrm{S}_{\mathrm{hh} / \mathrm{vv}}\right)$ (in $\mathrm{mm})$ the real part of the forward scattering amplitude at horizontal/vertical polarization, $\lambda$ the radar wavelength (in $\mathrm{mm}$, for the X-band radar wavelength used in this paper we have $\lambda=$ $33.3 \mathrm{~mm}$ ), $m$ the complex refractive index of water (a temperature of $20^{\circ} \mathrm{C}$ is taken for which $m=8.633+1.289 \mathrm{i})$. The scattering coefficients are obtained with the help of the Python PyTMatrix library (Leinonen 2014), which relies on the T-Matrix code (Mishchenko et al., 1996). The following set up is implemented for the computations: (i) an oblate spheroids model for drop shape is taken with an axis ratio - equivolumic diameter relation corresponding to the one implemented in the Parsivel ${ }^{2}$ rationale (Battaglia et al. 2010). In this model the ratio between the vertical and horizontal axis is equal to 1 for $D<1 \mathrm{~mm}, 0.7$ for $D$ $>5 \mathrm{~mm}$, and linearly related to $D$ with a slope equal to 0.075 in for intermediate $D$. (ii) Drop orientation is the same as in Leinonen et al. (2012); i.e. drops are partially aligned and a normal distribution (mean and standard deviation respectively equal to $0^{\circ}$ and $7^{\circ}$, in agreement with the findings of Bringi et al. 2008) characterizes the angle of the symmetry axis. Figure 1 displays typical curves of the scattering coefficients with respect to the particular diameter. As expected $\sigma_{B}$ is smaller for vertical polarization than horizontal ones and the differences get greater with increasing $D$ as drops get more oblate (Fig. 1.a). $\sigma_{B}$ vs. $D$ is plotted in $\log 10-\log 10$ plot in Fig. 1.b. It enables to point out a slight interest of using TMatrix computations code rather than rely on a pure Rayleigh approximation in which $\sigma_{B} \propto D^{6}$. This approximation remains valid for $D<3 \mathrm{~mm}$ (the linear regression yields a slope equal to 5.95), and a somewhat more complex behaviour is found for greater $D$. This transition occurs for decreasing drop diameter with decreasing wave length, meaning that it is more relevant to use T-Matrix computations instead of pure Rayleigh approximation at the Xband radar wave length than at the the C-band one. For instance instead of the $3 \mathrm{~mm}$ limit found here, the transition occurs at roughly $5 \mathrm{~mm}$ at a C-band radar wave length of $53.5 \mathrm{~mm}$ ).

The term used in the computation of $K_{\mathrm{dp}}$ (Eq. 2) exhibits a more complex behaviour with regards to drop equivolumic diameter (Fig. 1.c). Given the simplifying assumption made of spherical drops for $D<1 \mathrm{~mm}$, it theoretically yields a zero anisotropic scattering coefficient on this range and in fact a numerical noise (smaller than $10^{-16}$ software precision used), whereas prolate drops are usually expected (Beard and Chuang, 1987). This corresponds to a truncation of $K_{d p}$, with $D_{\min }=1$ that may introduce a bias for low intensity rainfalls to be discussed below. The small bump for drop ranging from 3 to $4 \mathrm{~mm}$ is followed by a power law-like behaviour for greater $D$ 's. The differential reflectivity $Z_{\mathrm{dr}}$ (no unit) is simply defined as the ratio between the horizontal and the vertical reflectivity: 


$$
Z_{d r}=\frac{Z_{h}}{Z_{v}}
$$

Observations are carried out with time steps of $30 \mathrm{~s}$. There is no missing data for these two months of observations for the Pasivel ${ }^{2} \mathrm{~s}$ and few ones for the PWS. In some sections of this paper, individual events are studied. An event is defined as a rainy period isolated by at least 15 minutes of dry condition before and after and resulting in more than $0.5 \mathrm{~mm}$ of total collected water depth.

The DSD is (at least partly) characterized with the help of the classical total drop concentration $N_{\mathrm{t}}\left(\mathrm{m}^{-3}\right)$ and the mass-weighted diameter $D_{\mathrm{m}}(\mathrm{mm})$ :

$$
\begin{aligned}
& N_{t}= \int_{D_{\min }}^{D_{\max }} N(D) d D \\
& D_{m}=\frac{\int_{D_{\min }}^{D_{\max }} N(D) D^{4} d D}{\int_{D_{\min }}^{D_{\max }} N(D) D^{3} d D}
\end{aligned}
$$

\section{2) Data for May and June 2016}

For better readability of this paper the results will be mainly presented for the device Pars\#2, and results for the other two will only be briefly mentioned. Figure 2 displays the temporal evolution of the rain rate for May and June 2016 and Pars\#2 data at the observation scale of $30 \mathrm{~s}$ and at the $5 \mathrm{~min}$ scale to which people are generally more familiar with. The cumulative depth is shown as well. $144 \mathrm{~mm}$ were recorded in May and $72 \mathrm{~mm}$ in June. The month of May was the wettest ever recorded in the Paris area. To provide order of magnitude, the average water depth in May is $63.2 \mathrm{~mm}$ in Paris (18.25 km West from disdrometer location) and 64.6 $\mathrm{mm}$ in Melun (32 km South from disdrometer location). The Month of June was also wetter than usual but with less extreme values. On average $49.6 \mathrm{~mm}$ are recorded in Paris and 53.9 in Melun (source http://www.meteofrance.com/climat/france)

Between 1 May 2016 and 31 June 2016, 50 events were recorded by the disdrometer resulting in a total water depth of $204 \mathrm{~mm}$. The number of events with total depth greater than 1, 2, 5, and $10 \mathrm{~mm}$ are respectively 42,24, 10 and 6 . The number of events with durations greater than $1,2,3,4$ and $12 \mathrm{~h}$ are respectively $40,23,14,8$ and 2 . The variability between the events of $N_{\mathrm{t}}$ and $D_{\mathrm{m}}$ is significant. The coefficient of variation for $N_{\mathrm{t}}$ is equal to $54 \%$ for a mean equal to $301 \mathrm{~m}^{-3}$. The coefficient of variation for $D_{\mathrm{m}}$ is equal to $42 \%$ for a mean equal to 1.39 $\mathrm{mm}$.

Figure 3 presents a scatter plot of the rain rates estimated at $30 \mathrm{~s}$ time steps of Pars\#1 and PWS vs. Pars\#2. It should be mentioned that there is some missing data during heavy rainfall for the PWS device resulting in roughly $7 \%$ of points missing. There is no missing data for both Pars\#1 and Pars\#2. Hence the analysis done in this paragraph is based only on the time steps when all devices are available to allow for a reliable comparison. For these time steps, the cumulative depth is of $181 \mathrm{~mm}$ for Pars\#1, $191 \mathrm{~mm}$ for Pars\#2 and $230 \mathrm{~mm}$ for PWS. It appears that the estimates for the two Parsivels are similar with a $6 \%$ split. The discrepancies with the PWS are larger, with $20 \%$ differences in terms of cumulative depths. These differences are great (it is more than $50 \%$ without the correction), and greater than the $10 \%$ noticed on a previous study (Gires et al. 2016). This could be related to the fact that both 
devices do not work on the same principle, i.e. one estimates drops' size and velocity from occlude light whereas this other uses refracted light.

\section{3) A focus on two different events}

Two events were selected to be studied more precisely throughout the paper. They exhibit different features:

(i) The 29-31 May 2016 event. It corresponds to the slow passing of a depression coming from the Atlantic Ocean (West of study area) that later went North-East. It started on 29-52016 at 19:56:00 (local times are provided) and lasted until 31-5-2016 at 15:5:30. It should be mentioned that there was a 1 h dry period on 30 May 2016 at approximately 5, but it is nevertheless presented as a single event. The main features for Pars\#2 are presented on Fig. 4. It is basically an event with limited rain rates, and a DSD centred on small drops (Fig. 4.c; $D_{\mathrm{m}}$ $=0.94 \mathrm{~mm}$ ). Its striking feature is its duration. Indeed it lasted more than $43 \mathrm{~h}$ (and almost 32 $\mathrm{h}$ without considering the first part) which is exceptionally long for this area. To stress this point authors carried out a short analysis on two 20 year long rainfall time series recorded by rain gauges located in the Val-de-Marne County less than $20 \mathrm{~km}$ from the disdrometer location (see Hoang et al. 2012 for a more detailed presentation of the data). For one rain gauge, the maximum observed duration for an event is only $13.2 \mathrm{~h}$, and the number of events with duration greater than $12 \mathrm{~h}, 10 \mathrm{~h}, 8 \mathrm{~h}$, and $6 \mathrm{~h}$ is respectively $2,4,7$, and 20 . The corresponding numbers for the other rain gauges are $1,1,3$, and 10, with a maximum duration of $12.8 \mathrm{~h}$.

(ii) The 17 June 2016 event. It was due to the presence of a cold and instable air mass over France that resulted in numerous storms all over the country. It started on 17-6-2016 at 17:36:00 (local times are provided) and lasted until 17-6-2016 at 19:05:00. It is a short event during which great rain rates (Fig. 5.a) were recorded (more than $70 \mathrm{~mm} . \mathrm{h}^{-1}$ at 30 second time steps and more than $40 \mathrm{~mm} \cdot \mathrm{h}^{-1}$ at $5 \mathrm{~min}$ time steps). No significant damage was reported on this area. The DSD exhibits a heavier tail (Fig. 5.c) with $D_{\mathrm{m}}=2.08 \mathrm{~mm}$. The number of large drops, i.e. with diameters greater than 3-4 mm, might be overestimated with the Parsivels, as this issue was recently reported by Park et al. (2017) for intense rainfall. The interpretation that it could be due to the orientation of the Parsivel sampling area with regards to the wind direction is discarded in this case because both perpendicular devices exhibit similar DSD. This possible overestimation could be due to nearly coincident drops intersecting the Parsivel laser causing multiple smaller drops to appear as a larger drop.

\section{4) Multifractal analysis of the data}

The variability across scales of the measured time series is quantified with the help of the theoretical framework of Universal Multifractals (UM) which has been extensively used to analyse and simulate geophysical fields exhibiting great variability over wide ranges of spatio-temporal scales (see Schertzer and Lovejoy 2011 for a recent review). Only the main elements are presented here, for more details readers are invited to refer to the review.

Let us consider a conservative field $\varepsilon_{\lambda}$ at resolution $\lambda$, defined as the ratio between the observation scale $l$ and the outer scale $L(\lambda=L / l)$. In practice the field measured at the maximum resolution is up-scaled (by averaging consecutive time steps) to obtain it at other resolutions. The first step of a multifractal analysis consists in computing the power spectra. If 
the field exhibits some scaling feature, then the spectra behaves as a power law with regards to the wave number $k$ :

$$
E(k) \propto k^{-\beta} \quad(6),
$$

where $\beta$ is the spectral slope.

If $\varepsilon_{\lambda}$ is a multifractal field, then its statistical moment orders scale with resolution as:

$\left\langle\varepsilon_{\lambda}{ }^{q}\right\rangle \approx \lambda^{K(q)}$

where $K(q)$ is the moment scaling function that fully characterizes the variability across scales of the field $\varepsilon_{\lambda}$. The quality of the scaling is assessed with the help of the Trace Moment (TM) analysis. It consists of plotting Eq. 7 in log-log; the slope of the obtained straight line being $K(q)$. In the specific framework of the Universal Multifractals (UM) toward which most multiplicative process converge (this a broad generalization of the central limit theorem, Schertzer and Lovejoy 1987, 1997), $K(q)$ is fully characterized with the help of only two scale invariant parameters $C_{1}$ and $\alpha . C_{1}$ is the mean intermittency co-dimension and measures the clustering of the (average) intensity at smaller and smaller scales $\left(C_{1}=0\right.$ for a homogeneous field). $\alpha$ is the multifractality index $(0 \leq \alpha \leq 2)$ and measures the clustering variability with regards to intensity level. In this paper, the Double Trace Moment (DTM) technique is used to estimate UM parameters (Lavallée et al. 1993).

If the studied field $\phi_{\lambda}$ appears to be non-conservative (as it is the case here), then a third parameter $H$, the non-conservation parameter, should be introduced. It is equal to zero for conservative fields. The TM and DTM analysis should be carried out on the conservative part $\varepsilon_{\lambda}$ of the field written as

$\phi_{\lambda}=\varepsilon_{\lambda} \lambda^{-H}$

And $H$ can be estimated with the help of (Tessier et al. 1993):

$$
\beta=1+2 H-K_{c}(2)
$$

Where $K_{\mathrm{c}}$ is the moment scaling function of the conservative part.

In practice TM and DTM techniques remain reliable as long as $H<0.5$. In case of greater $H$, they should be implemented not on $\phi_{\lambda}$, but on the underlying conservative field $\varepsilon_{\lambda}$. Theoretically, a fractional integration of order $H$ (equivalent to a multiplication by $k^{H}$ in the Fourier space) enables to compute $\varepsilon_{\lambda}$ from $\phi_{\lambda}$. Here we will use a common approximation (Lavallée et al. 1993) which consists of taking $\varepsilon_{\Lambda}$ at the maximum resolution simply equal to the renormalized absolute value of the fluctuations of the field i.e.:

$$
\varepsilon_{\Lambda}=\frac{\left|\phi_{\Lambda}(i+1)-\phi_{\Lambda}(i)\right|}{\left\langle\phi_{\Lambda}(i+1)-\phi_{\Lambda}(i) \mid\right\rangle}
$$

and then upscaling this field at other resolutions $\lambda$.

Analyses are carried out on ensemble average over various samples. Each sample is considered as a realisation of the process and is up-scaled independently. The same methodology as in Gires et al. 2016 is implemented to select the samples from the available data, i.e. "for each event (i) a sample size is chosen (a power of two, if possible); (ii) the maximum number of samples for this event is computed; (iii) the portion of the event of length equal to the sample size multiplied by the number of samples found in (ii) with the greatest cumulative depth is extracted; (iv) the extracted series is cut into various samples." 
Sample sizes of power of two is simpler to handle, but implies that some available data are actually not used. More precisely with sample sizes of 64, 128, 256, 512, 1024 and 2048 time steps, the percentage of data actually used of 90, 82, 64, 43, 26 and $11 \%$. Here a size of 64 time steps of $30 \mathrm{~s}$, corresponding to $32 \mathrm{~min}$ is used to maximise the amount of data used to estimate UM parameters and get more robust ones. Scaling features will also be discussed with other sample sizes.

UM analysis is mainly discussed for data collected with Pars\#2. The quality of the scaling on the available data is very good over the whole range of selected scales (30s - 32 min). See Fig. 6.a for the spectral analysis and 6.b for TM analysis where the quality of the linear regression is very good. Given the great value of the spectral slope (and $H$, see after), the TM and DTM analyses are implemented not on the field directly but on its. It should be mentioned that when tested with greater sample size, the scaling remains very good $\left(\mathrm{R}^{2}>0.99\right)$ up to 512 time steps $(\sim 4.3 \mathrm{~h})$. For sample size of 1024 (3 events and 5 samples), there seems to be a break also visible when performing the analysis with 2048 time steps. This limit of $4.3 \mathrm{~h}$ is a standard duration for meteorological situations in this area. Given the high quality of the scaling, the DTM analysis is reliable. The UM parameters found are: $\beta=1.98, C_{1}=0.19, \alpha=$ 1.64 and $H=0.65$. Similar results with same high quality of scaling are found for Pars\#1 with $\beta=2.08, C_{1}=0.19, \alpha=1.56$ and $H=0.70$. Due to the missing time steps it was not possible to carry out a similar analysis with the PWS data.

3) Radar relations at various temporal scales

\section{1) Presentation of the 3 relations used}

In this paper we study and discuss three radar relations (see references in the following section where parameters' values are discussed):

(i) The $Z_{h}-R$ relation: $Z_{h}=a R^{b}$

(ii) The $R-K_{d p}$ relation: $R=c K_{d p}{ }^{d}$

(iii) The $R-Z_{h}-Z_{d r}$ relation: $R=e Z_{h}{ }^{f} Z_{d r}{ }^{g}$

Here it should simply be mentioned that some authors express $Z_{d r}$ in $\mathrm{dB}$ (ex. Illingworth and Blackman 2002) while others keep it in linear scale (Leinonen 2012, Fuigueras i Ventura 2012) as we did here.

We focus on these relations because they are the most commonly used, especially the first two. Other ones can be found in the literature such as the $R-K_{d p}-Z_{d r}$ (Ryzhkov et al. 1995), but they are not explored here.

The parameters $a, b, c, d, e, f$, and $g$ are estimated using the measured DSD at the observation time step of $30 \mathrm{~s}$. For the $Z_{h}-R$ relation, an orthogonal regression is carried out for time steps with $R \geq 0.2 \mathrm{~mm} \cdot \mathrm{h}^{-1}$. An orthogonal regression minimizes the orthogonal distance from the data points to the fitted line, contrary to the ordinary linear regression which minimizes the vertical distance. For the $R-K_{d p}$ relation, an orthogonal regression is carried out for time steps with $K_{d p} \geq 0.2^{\circ} \cdot \mathrm{km}^{-1}$. For the $R-Z_{h}-Z_{d r}$ relation, a linear regression minimizing the quadratic error considering only the time steps with $R \geq 0.2 \mathrm{~mm} \cdot \mathrm{h}^{-1}$ is used. Computations are carried out only when more than 10 points (i.e. time steps) are available. 


\section{2) "Climatic" analysis}

First we consider all the available data to compute average values of the radar relations' parameters. In this study they will be denoted "climatic" values keeping in mind that they were obtained only with two months of data which is limited for such a statement. The plots yielding to the parameters' estimates of the $Z_{h}-R$ and $R-K_{d p}$ relations for the Pars\#2 data are shown in Fig. 7. The quality of the regression is good with coefficient of determination greater than 0.8. Estimates are reported in Table 1 for all radar relations and disdrometers. They are consistent with those commonly reported in the literature.

With regards to the $Z_{h}-R$ relationship we find $a=195$ and $b=1.78$ for Pars\#2. The value of $a$ is significantly greater for Pars\#1 with similar value of $b$. For PWS, values found for $a$ and $b$ are respectively greater and smaller than with Pars\#2 data. There is a wide variety of values reported in the literature for these parameters. Typical ones are $a=200$ and $b=1.6$ (the widely used parameter set since Marshal and Palmer, 1948) or $a=300$ and $b=1.4$ (which are the Nexrad standard values in USA, Fulton et al. 1998). Matrosov et al. (2002) found values of $a$ $=250$ and $b=1.68$ in the X-band using experimental raindrop size distributions. Jaffrain and Berne (2012b) reported values ranging from 220 to 320 for $a$ and from 1.4 to 1.6 for $b$ after analysing 36 rainfall events with disdrometer data. Hence it appears that the values found here for $a$ and $b$ are located respectively in the lower range and upper range of reported values in the literature.

This apparent slight discrepancy is actually not surprising. Indeed Verrier et al. (2013) showed that $a$ and $b$ are dependent on the scale at which they are computed. Given that they are computed here at $30 \mathrm{~s}$ which is much smaller than the minute or few minutes commonly used, the differences are expected. Values are in agreement with those found by Verrier et al. at this temporal scale. It should also be reminded that one should be careful when comparing values because they are also dependent on the regression method (mainly the choice to express quantity in log or not, and the regression type) used to extract them (Campos and Zawadski, 2000; Jaffrain and Berne 2012; Verrier et al. 2013).

For the $R-K_{d p}$ relation, we find $c=12.9$ and $d=0.75$ for Pars\#2. Very close estimates are found with Pars\#1 data and close ones with PWS data. Typical values for the X-band are $c=$ 19.9 and $d=0.85$ (Beard and Chuang, 1987) or $c=23.2$ and $d=0.79$ (Brandes at al. 2002). Jaffrain and Berne (2012b) found also in the X-band $c \sim[11-14]$ and $d \sim[0.69-0.75]$ according to the studied event. Leinonen (2012) found in the C-band $c=21.0 d=0.72$, meaning that the parameter $c$ would be similar to the one found here, had the study be done in X-band. Anagnostou et al. (2010) reported values of $c=19.26$ and $d=0.85$ in the X-band. Matrosov et al. (2002) found values of $c=12.3$ and $d=0.81$ in the X-band. Hence the values obtained in this analysis are in agreement with the ones found in the literature.

Finally we find for the $R-Z_{h}-Z_{d r}$ relation $e=0.023, f=0.77$ and $g=-4.05$ for Pars\#2. Comparable values are retrieved with the other devices. This relation is less used by the community than the other two. Leinonen et al. (2012) reported values of $e=0.012, f=0.822$ and $g=-2.28$. Ryzhkov et al. (2005) found $e \sim[0.0067-0.0159], f \sim[0.73-1]$ and $g \sim[-4.73$, $1.03]$ according to the drop shape model, and the type of data used (experimental or modelled DSD). 


\begin{tabular}{|l|l|l|l|l|}
\hline Radar relation & Parameter & Pars\#1 & Pars\#2 & PWS \\
\hline \multirow{2}{*}{$Z_{h}=a R^{b}$} & $\mathrm{a}$ & 243 & 195 & 240 \\
\cline { 2 - 5 } & $\mathrm{b}$ & 1.76 & 1.78 & 1.52 \\
\hline \multirow{2}{*}{$R=c K_{d p}{ }^{d}$} & $\mathrm{c}$ & 12.6 & 12.9 & 14.7 \\
\cline { 2 - 5 } & $\mathrm{d}$ & 0.80 & 0.75 & 0.82 \\
\hline \multirow{2}{*}{$R=e Z_{h}^{f} Z_{d r}^{g}$} & $\mathrm{e}$ & 0.018 & 0.023 & 0.012 \\
\cline { 2 - 5 } & $\mathrm{f}$ & 0.80 & 0.77 & 0.87 \\
\cline { 2 - 5 } & $\mathrm{g}$ & -3.95 & -4.05 & -4.52 \\
\hline
\end{tabular}

Table 1: "Climatic" parameters of the radar relations obtained with the disdrometer data used in this study.

\section{3) Event based analysis}

In this section the radar relations are estimated independently for each event with Pars\#2 data. Similar results are found for the other devices. They are computed only for events with more than 10 points available to carry out the linear regression and whose cumulative depth is greater than $3 \mathrm{~mm}$. These conditions result in 17 events studied for the $Z_{h}-R$ and $R-Z_{h}-Z_{d r}$ relations and 16 for the $R-K_{d p}$ relation. Figure 8.a displays $b$ vs $a$ for the $Z_{h}-R$ relation. No tendency was found between the event general features (duration, total rainfall amount...) and the parameters of the radar relations. It appears that the ranges of values is rather great and greater than the ones reported by Jaffrain and Berne (2012) in their analysis of 36 events over the Lausanne area (Switzerland). It is of the same order of magnitude as those found by Matrosov et al. (2002) who found $a \sim$ [94-624] and $b \sim$ [1.34-2.36] when analysing 15 rainfall events observed with a transportable $\mathrm{X}$-band radar. No trend is visible with this data. The variability is smaller for the exponent $b$ than for the pre-factor $a$, with coefficients of variation $(\mathrm{CV})$ respectively equal to $14 \%$ and 33\%. Figure 8.b displays $d$ vs. $c$ for the $R-K_{d p}$ relation. Again the range of obtained values is wider than in Jaffrain and Berne (2012b). Here there is no obvious trend between $c$ and $d$, maybe a slight positive correlation (as in Jaffrain and Berne 2012 reported) but nothing of a statistical significance. Similarly to the $Z_{h}-R$ relation, $C V$ is smaller for the exponent $d$ than the pre-factor $c$ (28\% vs. $41 \%$ ). The variability between events seems slightly greater for the parameters of the $R-K_{d p}$ relation than for the ones of the $Z_{h}-R$ relation. It is likely to be due to a greater sensitivity of the $R-K_{d p}$ relation than the $Z_{h}-R$ one to the disparities in terms of DSD. For these selected events we find $C V=35 \%$ for $D_{m}$ and $34 \%$ for $N_{t}$, which is only slightly smaller than the values found for all the events. Figure 8.c, 8.d and 8.e respectively display the $e(C V$ $=40 \%), f(C V=9.3 \%)$ and $g(C V=53 \%)$ parameters of the $R-Z_{h}-Z_{d r}$ relation vs. one another. There simply seems to be a negative correlation trend between $e$ and $f$. The $C V$ are greater than for the other relations except for $f$.

\section{4) Intra-event analysis}


In this section, we go a step further and investigate the variability of the $Z_{h}-R$ and $R-K_{d p}$ radar relations at the highest available temporal resolution with the help of data collected with Pars\#2 device. In order to achieve this, the parameters $a, b, c$ and $d$ are computed for each time step considering a moving window of 40 time steps (i.e. 10 minutes before and 10 minutes after). Again they are only computed if 10 points are available to perform the linear regression. This is done for the two selected events of 29-31 May 2016 and 17 June 2016.

With regards to the 29-31 May event, Fig. 9.a displays the computation of $a$ and $b\left(Z_{h}-R\right.$ relation) for the whole event. Figure 9.c and d shows the temporal evolution of the "local" (i.e. with the moving window) of respectively $a$ and $b$; while a scatter plot of $b$ vs. $a$ is in Fig. 9.b. Note that the colour of the points in Fig. 9.b corresponds to the time within the event at which $a$ and $b$ are estimated. It means that two points with similar colours correspond to the same moment of the storm. Figure 10 displays the same information but for the $R-K_{d p}$ radar relation. It should be noted that the number of time steps for which it is possible to compute $c$ and $d$ (only $6 \%$ of time steps for the May event) is more reduced than for $a$ and $b$. This is due to the condition $K_{d p} \geq 0.2^{\circ} \mathrm{km}^{-1}$ which requires significant rain rates. This explains the lack of information found on Fig. 10.c and 10.d. Figures 11 and 12 are the equivalent of respectively 8 and 9 but for the 17 June event. For this event the $R-K_{d p}$ can be fitted on most of the event.

It can be seen that at the event scale, the linear regression illustrating the radar relations are good meaning the underlying radar relations are reliable. Second it appears that the variability observed between events (see section 4.3) is also present within an event. Indeed the temporal evolutions of radar parameters exhibit some quick variability, even with the use of the moving window technique. Figure 13 displays the temporal evolution of $a$ and $b$ for the 29-31 May event for the three co-located disdrometers. It highlights that parameters $a$ and $b$ exhibit a very local variability, which if taken into account could improve rainfall retrieval with radars. Furthermore although with some differences (local variability, sampling variability, device functioning), it appears that similar patterns are found. It suggests that the temporal variability of $a$ and $b$ is associated with a variability of the underlying physical processes and not instrumental or sampling uncertainties. For the 17 June event a negative correlation between the parameters of a given radar relation ( $a$ and $b$ Fig 11.b or $c$ and $d$ Fig 12.b) is visible for the first 50 minutes. Such obvious correlations are not so visible for the May event which is longer. Let us note that for the $Z_{h}-R$ relation one can distinguish some correlation on small portions of the event (some "lines" on Fig. 9.b with similar colours); suggesting that such correlation would only be valid for short periods of time, typically few tens of minutes.

An attempt was made to establish a correlation between the mass weighted diameter $D_{\mathrm{m}}$ and the radar parameters. It was computed in a similar way with a moving window for each time step. Results are presented in Table 2. They are contrasted and do not enable to obtain strong conclusions. Limited correlation is found for the $Z_{h}-R$ relation. For the $R-K_{d p}$ an anticorrelation is noted with $c$, while contrasted results according to the event are found for $d$, suggesting a lower dependence on $D_{m}$. Similar results were found for Pars\#1.This issue should be further investigated to yield more robust conclusions. 


\begin{tabular}{|c|c|c|c|}
\hline \multicolumn{2}{|c|}{ Radar relation parameter } & $\begin{array}{c}29-31 \text { May } \\
2016\end{array}$ & $\begin{array}{c}17 \text { June } \\
2016\end{array}$ \\
\hline \multirow{2}{*}{$Z_{h}-R$} & $a$ & 0.34 & 0.069 \\
\cline { 2 - 4 } & $b$ & 0.60 & 0.15 \\
\hline \multirow{2}{*}{$R-K_{d p}$} & $c$ & -0.60 & -0.91 \\
\cline { 2 - 4 } & $d$ & -0.30 & 0.89 \\
\hline
\end{tabular}

Table 2: Correlation between the radar parameters and the mass weighted diameter $\left(D_{\mathrm{m}}\right)$ for the two selected events with Pars\#2

4) Consequences on rainfall retrievals with radars.

\section{1) Methodology}

In this section the portion of radar algorithms converting filtered radar quantities to rainfall rates are mimicked with the help of disdrometer measurements. More precisely:

(i) Pseudo-radar quantities $\left(Z, Z_{\mathrm{dr}}, K_{\mathrm{dp}}\right)$ are estimated with the help of the instantaneous DSD and Eqs. 1 and 2. The rain rate is computed from the raindrops collected.

(ii) The parameters of the radar relations are estimated at various temporal scales ("climatic", event, and local) with the help of log-regressions, as described in section 3.

(iii) Rain rates that would have been obtained with radars are simulated by implementing the radar relations. Four of them are tested; the three studied ones in previous sections and an additional hybrid one:

$-R_{r a d}=\left(\frac{Z_{h}}{a}\right)^{1 / b}$

- $R_{\text {rad }}=c K_{d p}^{d}$

- $R_{\text {rad }}=e Z_{h}{ }^{f} Z_{d r}{ }^{g}$

- A rather standard hybrid model which consists in using a $Z_{h}-R$ relation for low rain rates $\left(K_{d p} \leq 0.2^{\circ} \cdot \mathrm{km}^{-1}\right.$ ) and a $R-K_{d p}$ relation otherwise.

When "local" estimates of the radar relations' parameters are not available the "event" ones are used. When "event" estimates are not available, "climatic" ones are used. All computations are carried out at $30 \mathrm{~s}$ time steps. The issue of rain profiling algorithm using all the values along a full radar beam are not explored here (Anagnostou et al., 2004; Park et al., 2005; Testud et al. 1999) and only local algorithms are mimicked. These radar relations, which are used in all radar algorithms based on pre-defined scattering relations, are sensitive to the choice the drop shape model and the discrepancies with the unknown true shapes yield some significant uncertainty on the rain rate retrieval. It should be highlighted that the developed approach enables to artificially avoid this very complex problem by simply using the same to compute the reconstructed pseudo-radar quantities and the radar relations.

Obviously this cannot be the case with true radar data since the drop shape is then unknown.

With these algorithms, we obtain 12 (4 types with either "climatic", "event" or "local" parameters for the radar relations) pseudo-radar rainfall time series $\left(R_{\text {rad }}\right)$ for each event and they are compared with the rainfall time series obtained directly from drop measurements $(R)$. The first step of the comparison consists in performing the same multifractal analysis that was done in section 3 and checking whether similar results are obtained. In a more standard way, we also computed scores commonly used for such tasks (Diss et al., 2009; Emmanuel et al., 2012; Figueras I Ventura et al., 2012; Krajewski et al., 2010; Moreau et al. 2009): 
- The Normalized Bias $(N B)$ whose optimal value is $0: N B=\frac{\left\langle R_{r a d}\right\rangle}{\langle R\rangle}-1$

- The correlation coefficient (corr) which varies between -1 and 1 and whose optimal value is

1: corr $=\frac{\sum_{\forall i}\left(R_{i}-\langle R\rangle\right)\left(R_{r a d, i}-\left\langle R_{r a d}\right\rangle\right)}{\sqrt{\sum_{\forall i}\left(R_{i}-\langle R\rangle\right)^{2}} \sqrt{\sum_{\forall i}\left(R_{r a d, i}-\left\langle R_{r a d}\right\rangle\right)^{2}}}$

- The Nash-Sutcliffe model efficiency coefficient (Nash), which varies between $-\infty$ and 1

and whose optimal value is $1:$ Nash $=1-\frac{\sum_{\forall i}\left(R_{r a d, i}-R_{i}\right)^{2}}{\sum_{\forall i}\left(R_{i}-\langle R\rangle\right)^{2}}$

- The Root mean square error $(R M S E)$, which varies between 0 and $+\infty$ and whose optimal

value is $0: R M S E=\sqrt{\frac{\sum_{\forall i}\left(R_{r a d, i}-R_{i}\right)^{2}}{N}}$

- The Slope and Offset of the orthogonal linear regression. The optimal values are respectively 1 and 0.

Where $R_{\text {rad }}$ and $R$ correspond respectively to the reconstructed pseudo-radar data and rain drop derived rainfall. $<>$ denotes the average. Time steps (index $i$ in the previous formulas) of all the events are used in the sum for each indicator.

\section{2) Overall results}

Results are presented for the data collected with Pars\#2 device. Similar ones are found for the other disdrometers. Figure 14 displays the scatter plot of $R_{\text {rad }}$ Vs. $R$ for the 12 algorithms tested. Table 3 shows the output of the multifractal analysis performed on the various fields. For instance if we consider the $Z_{h}-R$ algorithm with "climatic" parameters, we find $\alpha=1.84$ and $C_{1}=0.25$ while for the actual series we have $\alpha=1.64$ and $C_{1}=0.19$. It means that the series obtained with the pseudo radar algorithm exhibits too strong multifractality and intermittency with regards to the actual one, resulting in unrealistic stronger extremes. Standard comparison scores are presented in Table 4. These results enable to sort the various algorithms in terms of performance.

Considering a fixed temporal scale for the computation of the radar relations' parameters (i.e. "climatic", "event" or "local") it appears that the hybrid algorithm is the one performing best. Indeed the UM parameters estimates are closer to actual values for the time series computed with this algorithm and the standard scores are better. This confirms the results found by Figueras i Ventura et al. (2012) who analysed at the hourly time scale radar data from France comparing them with rain gauge data. Then follow respectively the $R-Z_{h}-Z_{d r}$ and $Z_{h}-R$ algorithm. The behaviour of the $R-K_{d p}$ relation is trickier to analyse. Indeed in terms of scores, it seems to perform similarly or best than the other algorithms except for $N B$ and total cumulative depth. This is related to the behaviour for "small" intensity for which there is a strong negative bias. For instance with the climatic average, we have $N B$ equal to almost -0.3 for time steps when the rain rate is smaller than $10 \mathrm{~mm} \cdot \mathrm{h}^{-1}$ whereas it is lower than 0.1 with rain rates greater than $10 \mathrm{~mm} . \mathrm{h}^{-1}$ (which is visible on Fig. 14). Fitting it using the whole range 
of available rain rates yields very low regression quality. This is consistent with the more complex behaviour of the scattering coefficient for computing $K_{\mathrm{dp}}$ vs. $D$ (Fig. 1.c). Indeed the relation becomes clearer only for rather large drops which are visible during heavy rainfall periods, whereas the aforementioned truncation of drops with $D<1 \mathrm{~mm}$ cannot be forgotten for low intensity rainfalls

For each algorithm it appears that the performance improves when going from using "climatic" to "event" to "local" estimates of the radar relations' parameters. This effect was expected and is now quantified. The $Z_{h}-R$ algorithm is especially sensitive to this effect and its performance strongly improves when $a$ and $b$ parameters are tuned more locally. With "local" estimates, its performances become comparable with the $R-Z_{h}-Z_{d r}$ ones which is not the case with "climatic" estimates. Matrosov et al. (2002) noticed this effect for the $Z_{h}-R$ algorithm at the event scale. This improvement could somehow be expected from Fig. 1.a-b which displays the backscattering coefficient as a function of the equivolumic drop diameter. Indeed, the relation being straightforward, once parameters of the $Z_{h}-R$ are fitted with a local DSD which limits its influence on the variability (i.e. the main source of uncertainty), it is not surprising that the $Z_{h}-R$ performs well. This improvement is less pronounced for the hybrid algorithm mainly because its performances are already good with "climatic" estimates of radar parameters, and the hybrid algorithm somehow already tunes the parameters according to rain rates by using two different relations. Although it is practically complicated these results make a case for the use (when possible) of tuned radar relations' parameters, if not in real time at least during re-analysis. The rather good performances of the simple $Z_{h}-R$ algorithm show at first glance that when no dual-polarization radar can be installed, the combination of a disdrometer used to estimate in real time the parameters of the $Z_{h}-R$ relation and a single polarization radar can yield good rainfall estimates. However, one should keep in mind that tuned parameters rely on DSD measurement at a single point not taking into account its variability in space.

In this paper, multifractal techniques are used to assess the quality of pseudo-radar algorithms The closeness of UM parameters assessed on radar time series to the ones obtained on the actual rainfall time series may help to assess the performance of the algorithms. The use of such techniques is very interesting because it intrinsically validates the outcome across all studied scales and is not limited to a single resolution as it is the case for the standard scores also computed in this paper. Such practical applications of scaling techniques were already developed to assess the quality of high frequency long rainfall time series (Hoang et al. 2012). The analyses carried out here are another example. 


\begin{tabular}{|c|c|c|c|c|c|c|}
\hline \multicolumn{2}{|c|}{ Radar algorithm } & \multirow[t]{2}{*}{$\beta$} & \multirow[t]{2}{*}{$R^{2}$} & \multirow[t]{2}{*}{$\alpha$} & \multirow[t]{2}{*}{$C_{1}$} & \multirow[t]{2}{*}{$H$} \\
\hline $\begin{array}{l}\text { Parameter } \\
\text { estimation }\end{array}$ & $\begin{array}{c}\text { Radar } \\
\text { conversion }\end{array}$ & & & & & \\
\hline \multirow[t]{4}{*}{ Climatic } & $R_{r a d}=\left(\frac{Z_{h}}{a}\right)^{1 / b}$ & 1.69 & 0.98 & 1.84 & 0.25 & 0.59 \\
\hline & $R_{r a d}=c K_{d p}^{d}$ & 1.93 & 0.99 & 1.44 & 0.20 & 0.63 \\
\hline & $R_{r a d}=e Z_{h}^{f} Z_{d r}^{g}$ & 1.41 & 0.99 & 2.05 & 0.23 & 0.44 \\
\hline & Hybrid & 1.81 & 0.99 & 1.76 & 0.21 & 0.60 \\
\hline \multirow[t]{4}{*}{ Event } & $R_{r a d}=\left(\frac{Z_{h}}{a}\right)^{1 / b}$ & 1.58 & 0.99 & 1.79 & 0.24 & 0.51 \\
\hline & $R_{r a d}=c K_{d p}{ }^{d}$ & 1.97 & 0.99 & 1.42 & 0.19 & 0.63 \\
\hline & $R_{r a d}=e Z_{h}^{f} Z_{d r}^{g}$ & 1.55 & 0.99 & 1.91 & 0.22 & 0.49 \\
\hline & Hybrid & 1.97 & 0.99 & 1.60 & 0.19 & 0.65 \\
\hline \multirow[t]{4}{*}{ Local } & $R_{r a d}=\left(\frac{Z_{h}}{a}\right)^{1 / b}$ & 1.74 & 0.99 & 1.69 & 0.22 & 0.56 \\
\hline & $R_{r a d}=c K_{d p}{ }^{d}$ & 1.94 & 0.99 & 1.47 & 0.19 & 0.63 \\
\hline & $R_{r a d}=e Z_{h}^{f} Z_{d r}^{g}$ & 1.82 & 0.99 & 1.72 & 0.20 & 0.59 \\
\hline & Hybrid & 1.96 & 0.99 & 1.61 & 0.19 & 0.65 \\
\hline \multicolumn{2}{|c|}{ For the actual time series } & 1.98 & 0.99 & 1.64 & 0.19 & 0.65 \\
\hline
\end{tabular}

Table 3: Results of the multifractal analysis performed on the rain rates computed via reconstructed pseudo-radar data and algorithms $\left(R_{\text {rad }}\right)$ vs. the one obtained from direct drop measurements $(R)$ for the data collected with the help of Pars\#2 device. 


\begin{tabular}{|c|c|c|c|c|c|c|c|c|}
\hline \multicolumn{2}{|c|}{ Radar algorithm } & \multirow[t]{2}{*}{$N B$} & \multirow[t]{2}{*}{ corr } & \multirow[t]{2}{*}{ Nash } & \multirow[t]{2}{*}{$R M S E$} & \multirow[t]{2}{*}{ Slope } & \multirow[t]{2}{*}{ Offsett } & \multirow{2}{*}{$\begin{array}{l}\text { Cumul } \\
\text { (mm) }\end{array}$} \\
\hline $\begin{array}{l}\text { Parameter } \\
\text { estimation }\end{array}$ & $\begin{array}{c}\text { Radar } \\
\text { conversion }\end{array}$ & & & & & & & \\
\hline \multirow[t]{4}{*}{ Climatic } & $R_{r a d}=\left(\frac{Z_{h}}{a}\right)^{1 / b}$ & 0.11 & 0.85 & -0.68 & 22.7 & 1.7 & -1.2 & 229 \\
\hline & $R_{r a d}=c K_{d p}^{d}$ & -0.24 & 0.96 & 0.88 & 1.67 & 1.05 & -0.58 & 153 \\
\hline & $R_{r a d}=e Z_{h}^{f} Z_{d r}^{g}$ & -0.065 & 0.86 & 0.69 & 4.2 & 0.90 & -0.07 & 192 \\
\hline & Hybrid & -0.032 & 0.94 & 0.85 & 2.0 & 1.0 & -0.15 & 200 \\
\hline \multirow[t]{4}{*}{ Event } & $R_{r a d}=\left(\frac{Z_{h}}{a}\right)^{1 / b}$ & 0.016 & 0.90 & 0.73 & 3.6 & 1.1 & -0.08 & 209 \\
\hline & $R_{r a d}=c K_{d p}^{d}$ & -0.100 & 0.95 & 0.89 & 1.5 & 0.89 & 0.02 & 183 \\
\hline & $R_{r a d}=e Z_{h}^{f} Z_{d r}^{g}$ & -0.017 & 0.89 & 0.70 & 4.1 & 1.1 & -0.19 & 201 \\
\hline & Hybrid & -0.033 & 0.96 & 0.92 & 1.0 & 0.89 & 0.15 & 199 \\
\hline \multirow[t]{4}{*}{ Local } & $R_{r a d}=\left(\frac{Z_{h}}{a}\right)^{1 / b}$ & 0.002 & 0.94 & 0.88 & 1.6 & 0.96 & -0.09 & 206 \\
\hline & $R_{r a d}=c K_{d p}^{d}$ & -0.098 & 0.96 & 0.92 & 1.1 & 0.96 & -0.11 & 183 \\
\hline & $R_{r a d}=e Z_{h}^{f} Z_{d r}^{g}$ & -0.011 & 0.96 & 0.92 & 1.1 & 0.98 & -0.06 & 202 \\
\hline & Hybrid & -0.008 & 0.98 & 0.96 & 0.48 & 0.96 & -0.06 & 204 \\
\hline
\end{tabular}

Table 4: Standard scores of the rain rates computed via reconstructed pseudo-radar data and algorithms $\left(R_{\mathrm{rad}}\right)$ vs. the one obtained from direct drop measurements $(R)$ (total cumulative depth $=204 \mathrm{~mm}$ ) for the data collected with the help of Pars\#2 device.

\section{3) Focus on the two selected events}

In this section, we illustrate more precisely the general results discussed quantitatively in the previous section on the performance of the various radar algorithms. This is done by looking into more details at the time series for the two selected events and Pars\#2 data.

Figure 15.a displays the temporal evolution of the rain rates obtained via reconstructed pseudo-radar data and the three $Z_{h}-R$ algorithms ("climatic", "event", and "local") along with the direct estimation of $R$. The cumulative depth is shown in Fig. 15.b, where it is visible that the "climatic" estimates yield the worst cumulative depth (an underestimation in this case). A closer look at the temporal evolution of rain rates confirms the improvement brought by the "local" (in time) estimates of the radar relations' parameters. For example during the pseudo-peak slightly before $4.7 \mathrm{~h}$ (Fig. 15.c) the rain rate is below $5 \mathrm{~mm} \cdot \mathrm{h}^{-1}$ and the use of either the "climatic" or "event" relations yields strong overestimation, while the local parameters enable to better represent actual rain rates. Similar conclusion is found around the peak at $11.9 \mathrm{~h}$, with an underestimation by the "climatic" and "event" based algorithms. The hybrid models yield similar results, but results are not shown here because of the actually limited number of time steps for which a local $R-K_{d p}$ can be computed (6\%).

Figure 16 (left column) displays the temporal evolution of the rain rates computed via reconstructed pseudo-radar data and the 4 algorithms with "climatic", "event", and "local" parameters along with the direct estimation of $R$. Figure 16 (right column) exhibits the corresponding cumulative depth. Again the "local" estimates of radar relations' parameter 
provide the best estimates. In this case the "climatic" parameters yield an overestimation expect for $R-Z_{h}-Z_{d r}$ algorithm. It is especially visible for the $Z_{h}-R$ algorithm. The improvement brought by the "event" based and "local" estimates are visible on the successive peaks (Fig. 16.a).

\section{5) Conclusion}

In this paper, the rainfall data measured by disdrometers of Ecole des Ponts ParisTech during the two extremely wet months of May and June 2016 are analysed. At first, this rainfall data exhibit very good quality of scaling behaviour over scales ranging from $30 \mathrm{~s}$ to few hours.

Classical relations between rain rate and the horizontal reflectivity, the differential phase shift and the differential reflectivity $\left(Z_{h}-R, R-K_{d p}, R-Z_{h}-Z_{d r}\right.$ ) are investigated with the help of pseudo-radar quantities reconstructed from the disdrometer data. This reconstruction requires that both the DSD and the drop centre distribution are homogeneous. In this simplistic framework, the characteristic parameters of these power-law relations are computed at various scales: "climatic", "event" and "local" with moving time window. It appears that these parameters exhibit a strong variability from one event to the other and even within an event.

Finally these radar relations are used to reconstruct rain rate time series that would be observed with the help of weather radars. In addition to the previously mentioned relations, the standard hybrid model relying on a $Z_{h}-R$ relation for low intensities and a $R-K_{d p}$ relation for large intensities is tested. An innovative methodology based on assessing the quality of an algorithm on its ability to reproduce the scaling behaviour of actual time series was developed. This ensures that results are valid across scales. The main conclusion is that the hybrid algorithm is the one performing best for the pseudo-radar quantities, whatever the temporal scale of the computation of the radar relations' parameters. However, the disdrometer algorithm estimating the drop shape unfortunately truncates the computation of the pseudo $K_{d p}$ at $D_{\min }=1 \mathrm{~mm}$ and therefore the contribution of the prolate drops. It also appears that the performances of all algorithms significantly improve by using parameters computed over shorter period of time, but this unfortunately corresponds to less predictive value. Not too surprisingly, the famous $Z_{h}-R$ relation tuned with "local" parameter estimates exhibits very good performance, but this seems to be a somewhat artificial result from the power-law-like behaviour of the radar cross-section $\sigma(D)$. Overall, the analyses presented in this paper call for extended studies that would take into account (including in real time) the spatial variability of the DSD and the possible clustering of drops, as well as the contribution of the prolate drops, that could not be addressed in this paper.

\section{Acknowledgements}

Authors greatly acknowledge partial financial support form the Chair "Hydrology for Resilient Cities" (endowed by Veolia) of Ecole des Ponts ParisTech, 
References

[1] Anagnostou, E., Anagnostou, M., Krajewski, W., Kruger, A., Miriovsky, B., 2004. HighResolution Rainfall Estimation from X-Band Polarimetric Radar Measurements. J. Hydrometeor. 5, 110-128, doi: 10.1175/1525-7541(2004)005<0110:HREFXP>2.0.CO;2.

[2] Anagnostou, M., Kalogiros, J., Anagnostou, E., Tarolli, M., Papadopoulos, A., Borga, M., 2010. Performance evaluation of high-resolution rainfall estimation by X-band dualpolarization radar for flash flood applications in mountainous basins. J. Hydrol, 394 (1-2), 416, http://dx.doi.org/10.1016/j.jhydrol.2010.06.026.

[3] Battaglia, A., Rustemeier, E., Tokay, A., Blahak, U., Simmer, C., 2010. PARSIVEL Snow Observations: A Critical Assessment. Journal of Atmospheric and Oceanic Technology, 27(2), 333-344.

[4] Beard KV, Chuang C. 1987. A new model for the equilibrium shape of raindrops. J. Atmos. Sci. 44: 1509-1524.

[5] Brandes, E.A., Zhang, G., Vivekanandan, J., 2002. Experiments in rainfall estimation with a polarimetric radar in a subtropical environment. J. Appl. Meteorol.,41, 674-685.

[6] Bringi, V.N., Thurai, M. and Brunkow, D.A., 2008. Measurements and inferences of raindrop canting angles. Electronics Letters, 44(24), 1425-1426.

[7] Campbell Scientific Ltd, 2012. PWS100 Present Weather Sensor, User Guide.

[8] Campos, E., Zawadzki, I., 2000. Instrumental Uncertainties in Z-R Relations. Journal of Applied Meteorology, 39(7), 1088-1102.

[9] Diss, S., Testud, J., Lavabre, J., Ribstein, P., Moreau, E., Parent du Chatelet, J., 2009. Ability of a dual polarized X-band radar to estimate rainfall. Adv. Water Resour. 32 (7), 975985.

[10] Ellis, R.A., Sandford A.P., Jones G.E. , Richards J. , Petzing, J., Coupland, J.M., 2006. New laser technology to determine present weather parameters. Measurement Science \&Technology, 17 (7), 1715-1722. doi:10.1088/0957-0233/17/7/009.

[11] Emmanuel, I., Andrieu, H. and Tabary, P., 2012. Evaluation of the new French operational weather radar product for the field of urban hydrology. Atmospheric Research 103, 20-32.

[12] Figueras i Ventura, J., Boumahmoud, A.-A., Fradon, B., Dupuy, P., Tabary, P., 2012. Long-term monitoring of French polarimetric radar data quality and evaluation of several polarimetric quantitative precipitation estimators in ideal conditions for operational implementation at C-band. Q. J. R. Meteorol. Soc. 138 (669), 2212-2228.

[13] Fulton, R.A., Bredienbach, J.P., Seo D.-J., Miller, D.A., O'Bannon, T., 1998. The WSR88 rainfall algorithm. Weather Forecasting. 13: 377-395. 
[14] Gires, A., Tchiguirinskaia, I., Schertzer, D., 2016. Multifractal comparison of the outputs of two optical disdrometers. Hydrological Sciences Journal,

DOI:10.1080/02626667.2015.1055270.

[15] Hoang, C.T., Tchiguirinskaia, I., Schertzer, D., Arnaud, P., Lavabre, J., Lovejoy S., 2012. Assessing the high frequency quality of long rainfall series. Journal of Hydrology, 438$439,39-51$.

[16] Illingworth, A., Blackman, T., 2002. The Need to Represent Raindrop Size Spectra as Normalized Gamma Distributions for the Interpretation of Polarization Radar Observations. J. Appl. Meteor. 41, 286-297, doi: 10.1175/1520-0450(2002)041<0286:TNTRRS>2.0.CO;2.

[17] Jaffrain, J., Berne, A., 2012a. Influence of the Subgrid Variability of the Raindrop Size Distribution on Radar Rainfall Estimators. J. Appl. Meteorol. Climatol. 51(4), 780-785.

[18] Jaffrain, J., Berne, A., 2012b. Quantification of the small-scale spatial structure of the raindrop size distribution from a network of disdrometers. J. Appl. Meteorol. Climatol. 51 (5), 941-953.

[19] Krajewski, W.F., Villarini, G., Smith, J.A., 2010. Radar - Rainfall uncertainties : Where are We after Thirty Years of Effort? Bulletin of the American Meteorological Society 91 (1), 87-94.

[20] Lavallée, D., Lovejoy, S., Ladoy, P., 1993. Nonlinear variability and landscape topography: analysis and simulation. In: L. de Cola and N. Lam (Editors), Fractas in geography. Prentice-Hall, pp. 171-205.

[21] Leinonen, J., Moisseev, D., Leskinen, M., Petersen, W.A., 2012. A Climatology of Disdrometer Measurements of Rainfall in Finland over Five Years with Implications for Global Radar Observations. J. Appl. Meteorol. Climatol. 51(2), 392-404.

[22] Leinonen, J., 2014. High-level interface to T-matrix scattering calculations: architecture, capabilities and limitations. Optics Express. 22(2), 1655-1660.

[23] Loffler-Mang, M., Joss, J., 2000. An Optical Disdrometer for Measuring Size and Velocity of Hydrometeors. Journal of Atmospheric and Oceanic Technology 17(2), 130-139.

[24] Lovejoy, S., Duncan, M.R., Schertzer, D., 1996. Scalar multifractal radar observers problem. J. Geophys. Res. 101, 26479-26492.

[25] Marshall, J. S., and W. M. Palmer (1948). The distribution of raindrops with size, J. Meteor., 5, 165-166.

[26] Matrosov, S., Clark, K., Martner, B., Tokay, A., 2002. X-Band Polarimetric Radar Measurements of Rainfall. J. Appl. Meteor. 41, 941-952, doi: 10.1175/15200450(2002)041<0941:XBPRMO>2.0.CO;2.

[27] Mishchenko, M.I., Travis, L.D. and Mackowski, D.W., 1996. T-matrix computations of light scattering by nonspherical particles: A review. Journal of Quantitative Spectroscopy and Radiative Transfer, 55(5), 535-575. 
[28] Moreau, E., Testud, J. and Le Bouar, E., 2009. Rainfall spatial variability observed by Xband weather radar and its implication for the accuracy of rainfall estimates. Advances in Water Resources 32 (7), 1011-1019.

[29] OTT, 2014. Operating instructions, Present Weather Sensor OTT Parsivel2.

[30] Park, S., Bringi, V., Chandrasekar, V., Maki, M., Iwanami, K., 2005. Correction of Radar Reflectivity and Differential Reflectivity for Rain Attenuation at X Band. Part I: Theoretical and Empirical Basis. J. Atmos. Oceanic Technol. 22, 1621-1632, doi: 10.1175/JTECH1803.1.

[31] Park, S., H. Kim, Y. Ham, and S. Jung, 2017. Comparative Evaluation of the OTT PARSIVEL2 Using a Collocated Two-Dimensional Video Disdrometer. Journal of Atmospheric and Oceanic Technology 2017 34:9, 2059-2082, https://doi.org/10.1175/JTECHD-16-0256.1

[32] Ryzhkov, A.V., Zrnic D.S., 1995. Comparison of dual-polarization radar estimators in rain. J. Atmos. Oceanic Technol. 12, 249-256.

[33] Ryzhkov, A., Giangrande, S., Schuur, T. 2005. Rainfall Estimation with a Polarimetric Prototype of WSR-88D. J. Appl. Meteor. 44, 502-515, doi: 10.1175/JAM2213.1.

[34] Schertzer, D., Lovejoy, S., 1987. Physical modelling and analysis of rain and clouds by anisotropic scaling and multiplicative processes. J. Geophys. Res. 92(D8), 9693-9714.

[35] Schertzer, D., Lovejoy, S., 1997. Universal multifractals do exist!: Comments. Journal of Applied Meteorology 36(9), 1296-1303.

[36] Schertzer, D., Lovejoy, S., 2011. Multifractals, generalized scale invariance and complexity in geophysics. International Journal of Bifurcation and Chaos 21(12), 3417-3456.

[37] Schertzer, D., Tchiguirinskaia, I., Lovejoy, S., 2012. Getting higher resolution rainfall estimates: X-band radar technology and multifractal drop distribution. Proceedings of the Weather Radar and Hydrology Symposium Held in Exeter, UK, April 2011. IAHS Publ. Vol. 351.

[38] Tessier, Y., Lovejoy, S., Schertzer, D., 1993. Universal Multifractals: theory and observations for rain and clouds. Journal of Applied Meteorology 32(2), 223-250.

[39] Testud J., Le Bouar, E., Obligis E., Ali-Mehenni M., 1999. The rain profiling algorithm applied to polarimetric weather radar, Jour. Atmosph. and Ocean. Technol.17 332-356.

[40] Verrier, S., Barthès, L., Mallet, C., 2013. Theoretical and empirical scale dependency of Z-R relationships: Evidence, impacts, and correction. J. Geophys. Res. Atmos. 118, 74357449, doi:10.1002/jgrd.50557. 
Figures:

(a)

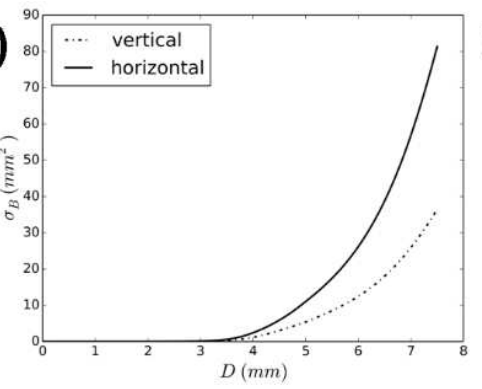

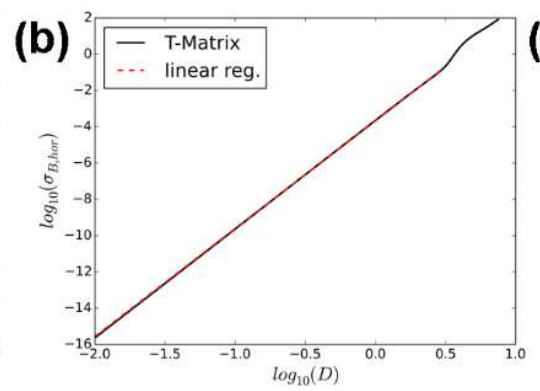

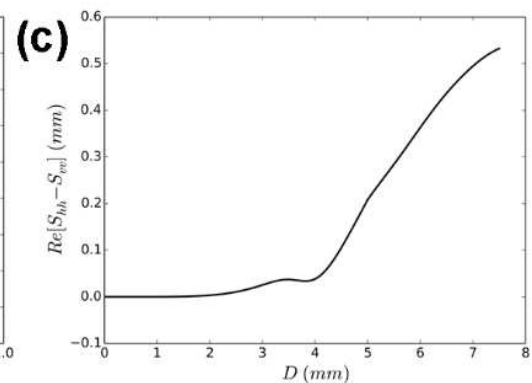

Figure 1: Scattering coefficients with respect to particular diameter (a) $\sigma_{B ; h, v}$ vs. $D$ (b) $\sigma_{B ; h}$ vs. $D$ in $\log -\log$ plot along with a linear regression for $D<3 \mathrm{~mm}(\mathrm{c}) \operatorname{Re}\left[S_{h h}(D)-S_{v v}(D)\right]$ vs. $D$

\section{May 2016}
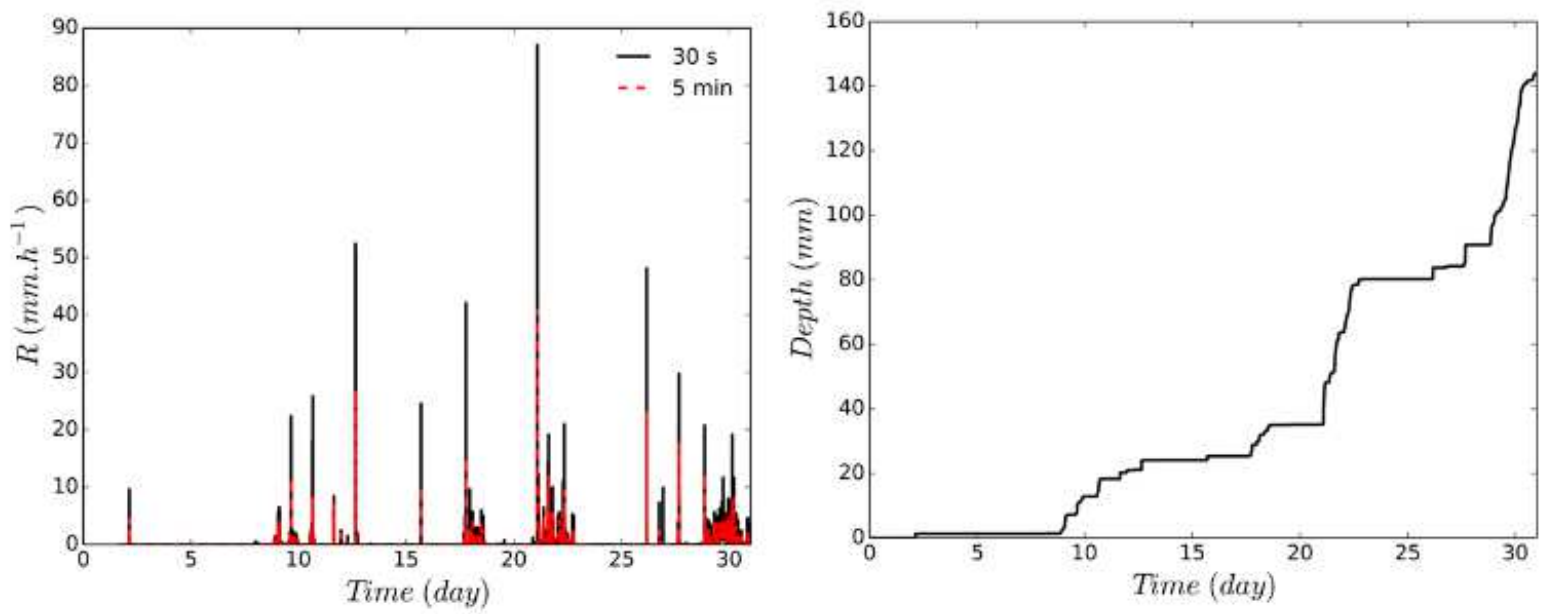

June 2016
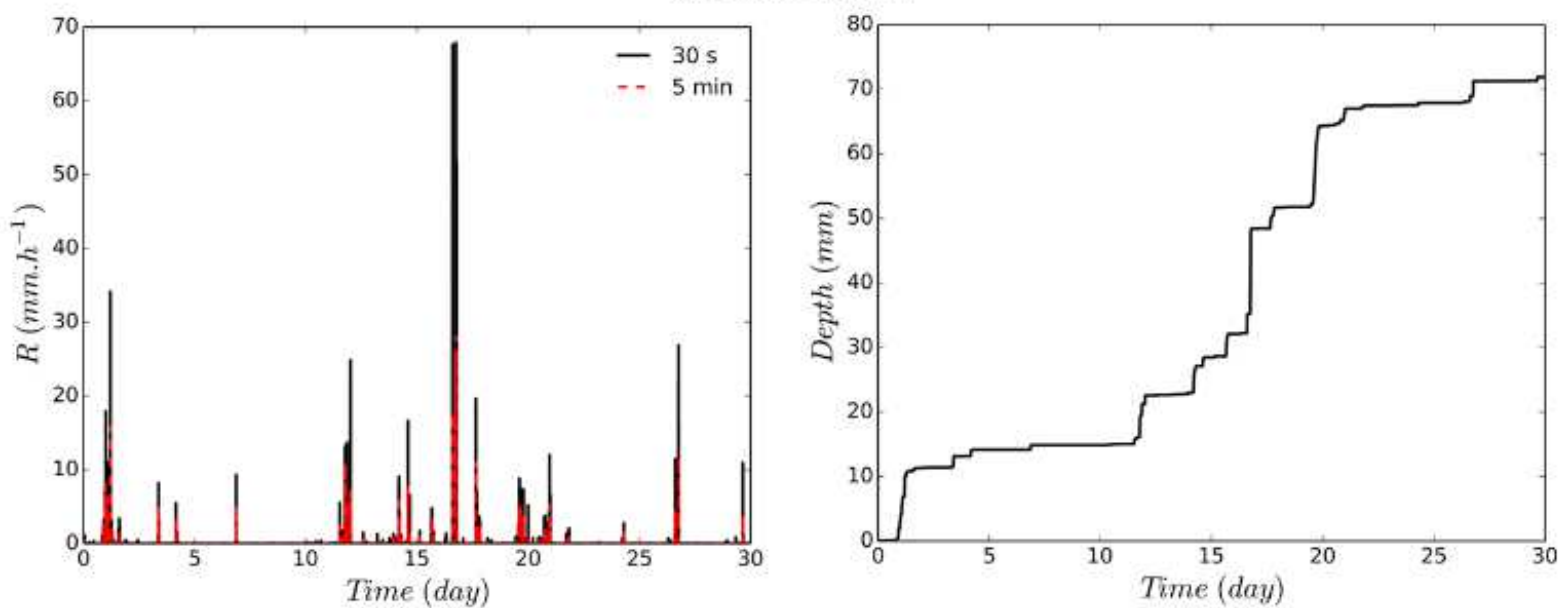

Figure 2: (Left) Temporal evolution of the rain rate in $\mathrm{mm} . \mathrm{h}^{-1}$ with $30 \mathrm{~s}$ time steps (solid black, the observation time step) and 5 min time steps (dashed red). (Right) Cumulative rainfall depth in mm. The months of May (top) and June (bottom) are presented separately. 

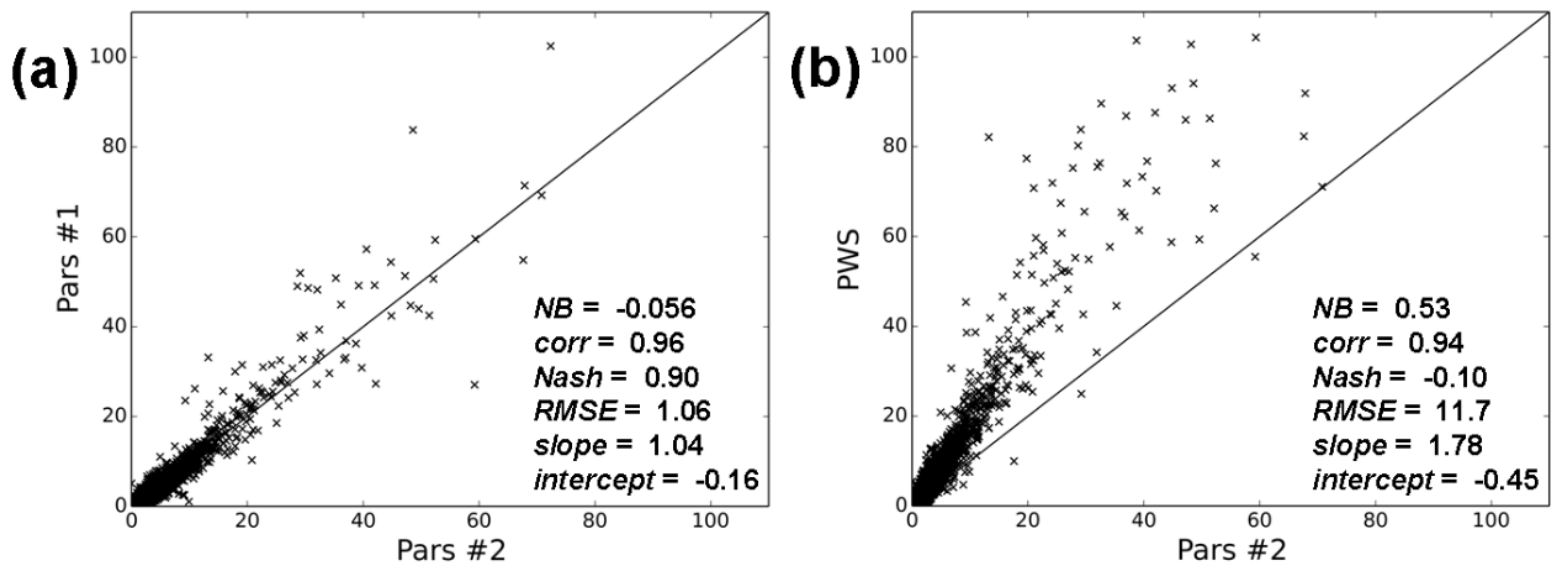

Figure 3: (a) Scatter plot of the 30 s time step rain rate for Pars\#1 vs. Pars\#2. (b) Same as (a) but for PWS vs. Pars\#2
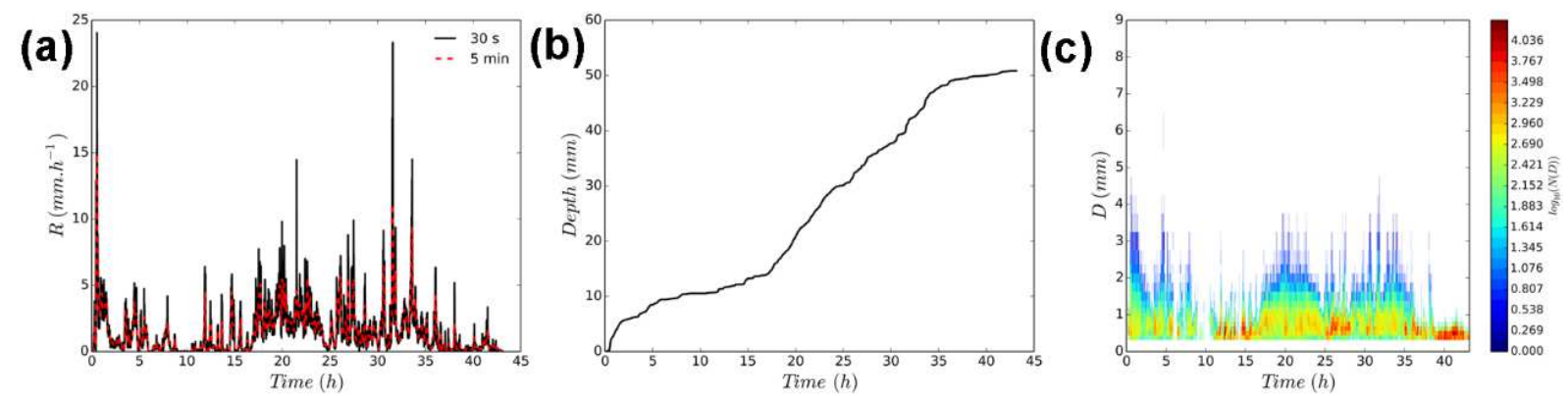

Figure 4: For the 29-31 May 2016 event with device Pars\#2. (a) Temporal evolution of the rain rate in mm. $\mathrm{h}^{-1}$ with $30 \mathrm{~s}$ time steps (solid black, the observation time step) and 5 min time steps (dashed red). (b) Cumulative rainfall depth in $\mathrm{mm}$. (c) Temporal evolution of the DSD $N(D)$.
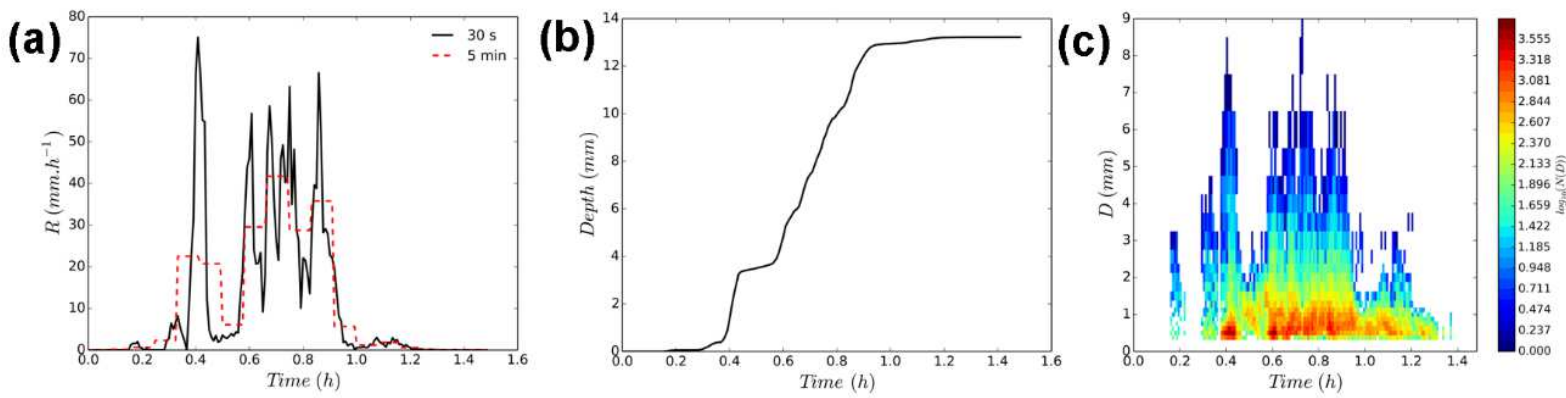

Figure 5: For the 17 June 2016 event with device Pars\#2. (a) Temporal evolution of the rain rate in $\mathrm{mm} . \mathrm{h}^{-1}$ with $30 \mathrm{~s}$ time steps (solid black, the observation time step) and 5 min time steps (dashed red). (b) Cumulative rainfall depth in $\mathrm{mm}$. (c) Temporal evolution of the DSD $N(D)$. 

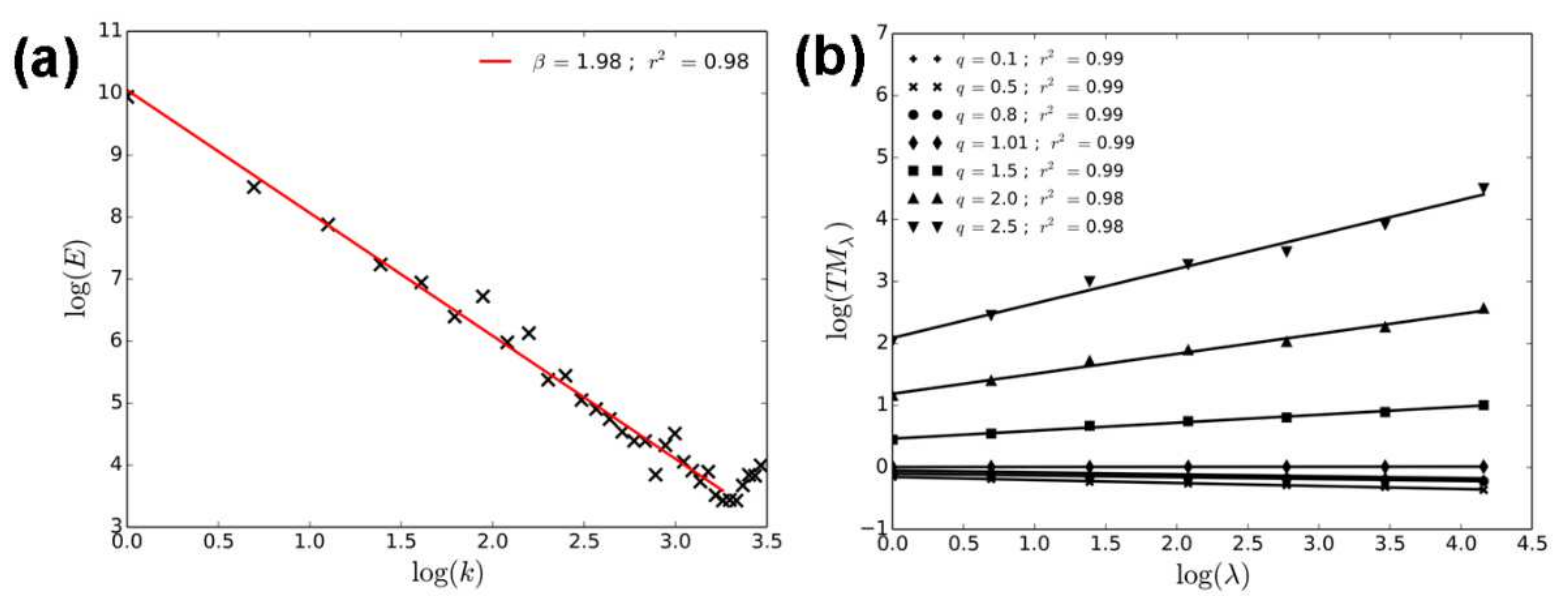

Figure 6: For the data collected with Pars\#2 (a) Spectral analysis, i.e. Eq. 6 in log-log (b) TM analysis, i.e. Eq. 7 in log-log. Samples of size 64, corresponding to $32 \mathrm{~min}$, are used.
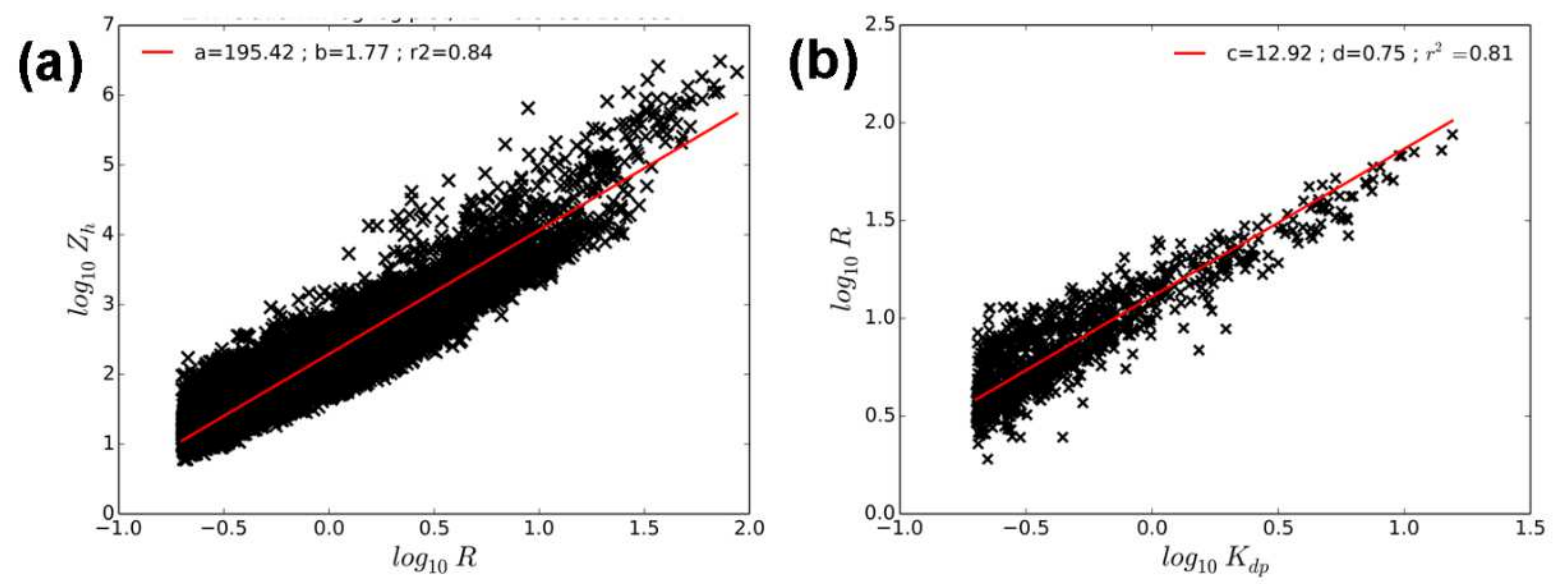

Figure 7: For the Pars\#2 device, estimation of the exponents of the power-law relations $Z_{h}-R$ (a) and $R-K_{d p}$ (b) by regression in a log-log plot, considering all the available events. 

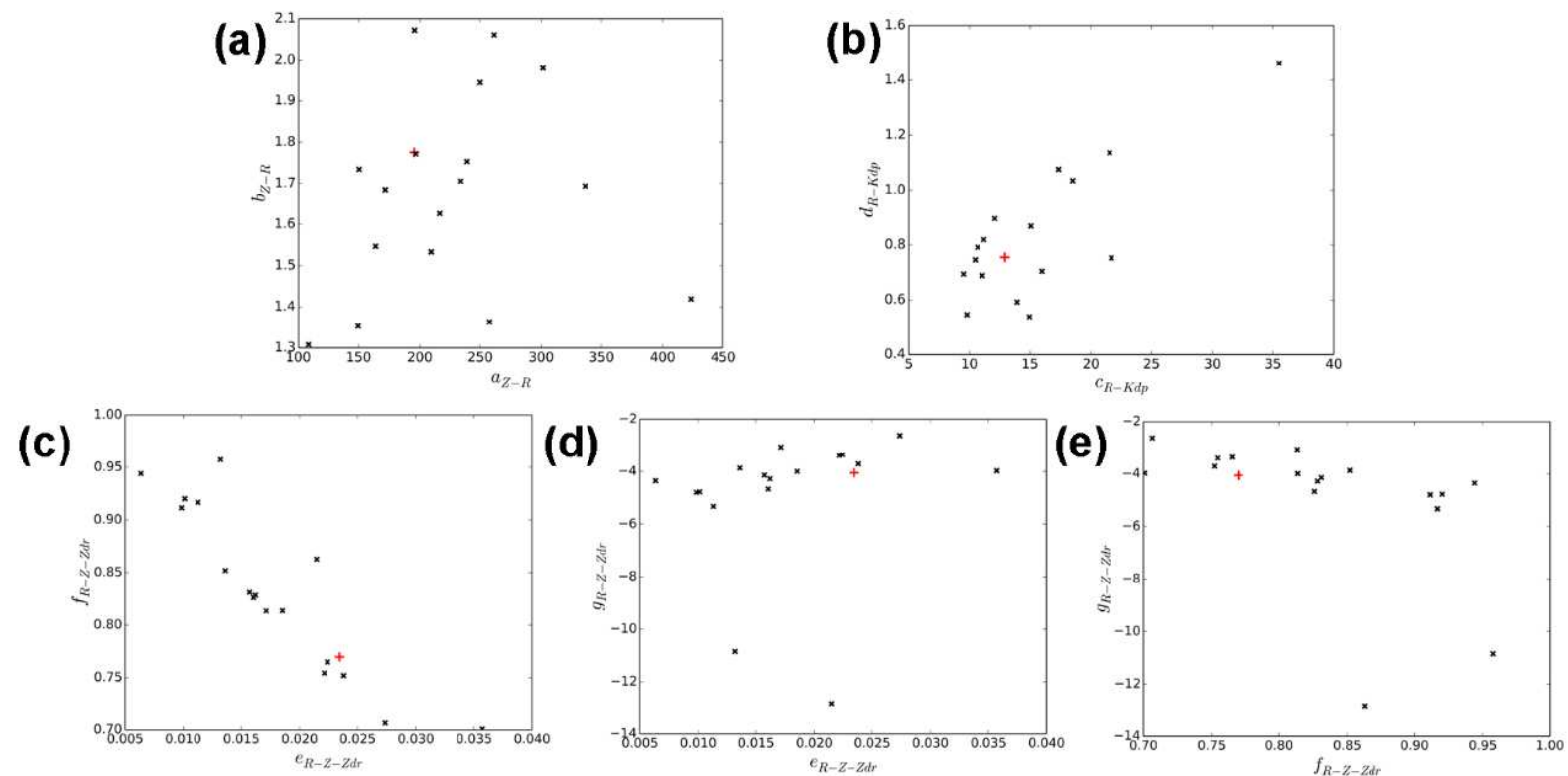

Figure 8: For the Pars\#2 device, plot of the radar relations' exponents vs. one another for the $Z_{h}-R$ (a), the $R-K_{d p}$ (b) and the $R-Z_{h}-Z_{d r}$ (c, d, e) power-law relations. The red crosses represent the values obtained by fitting the relations with the entire data set.

(a)

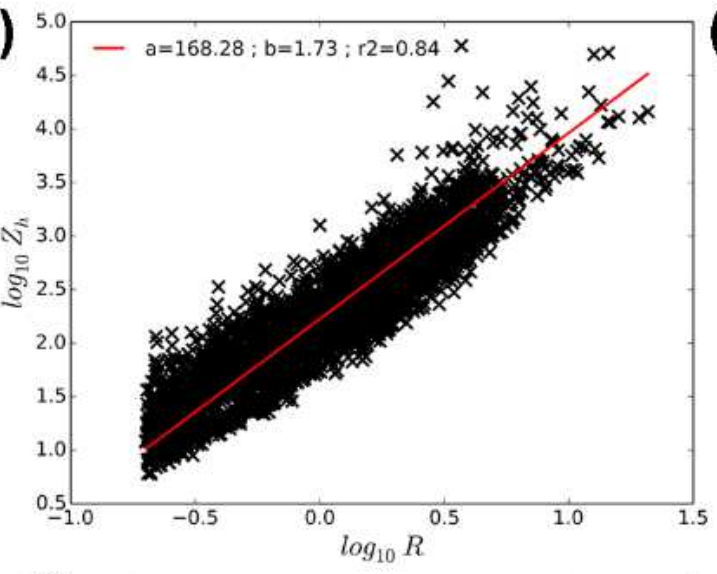

(b)

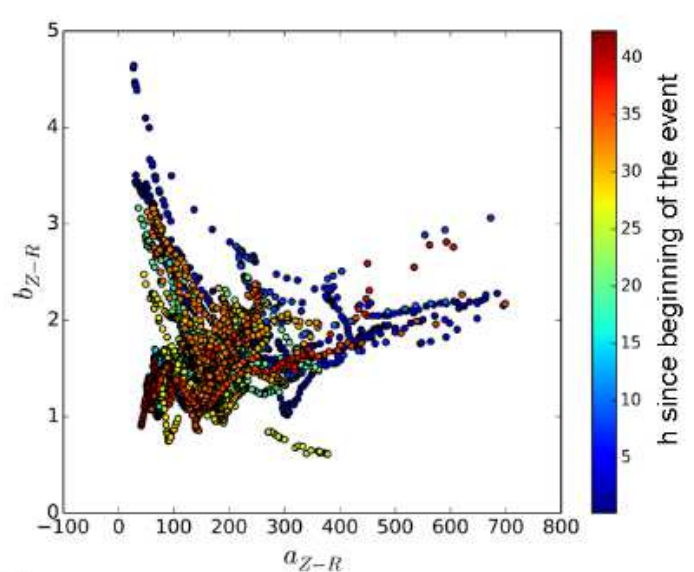

(d)

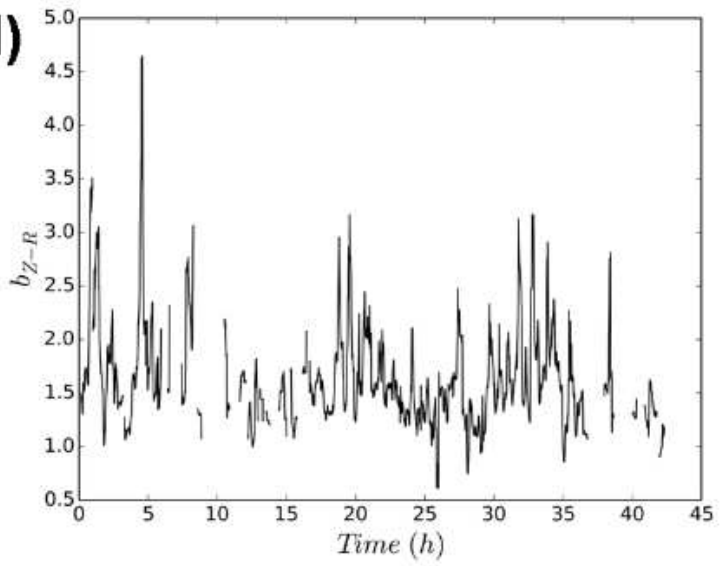

Figure 9: $Z_{h}-R$ relation for the 29-31 May 2016 event with Pars\#2. (a) Computation of the parameters $a$ and $b$ at the event scale (eq. 10 in a log-log plot). (b) Scatter plot of $b$ vs. $a$ estimated for each time step (20 minutes moving window). The colour of the points corresponds to the time within the event at which the parameters are estimated (c) Temporal evolution of $a$. (d) Temporal evolution of $b$. 

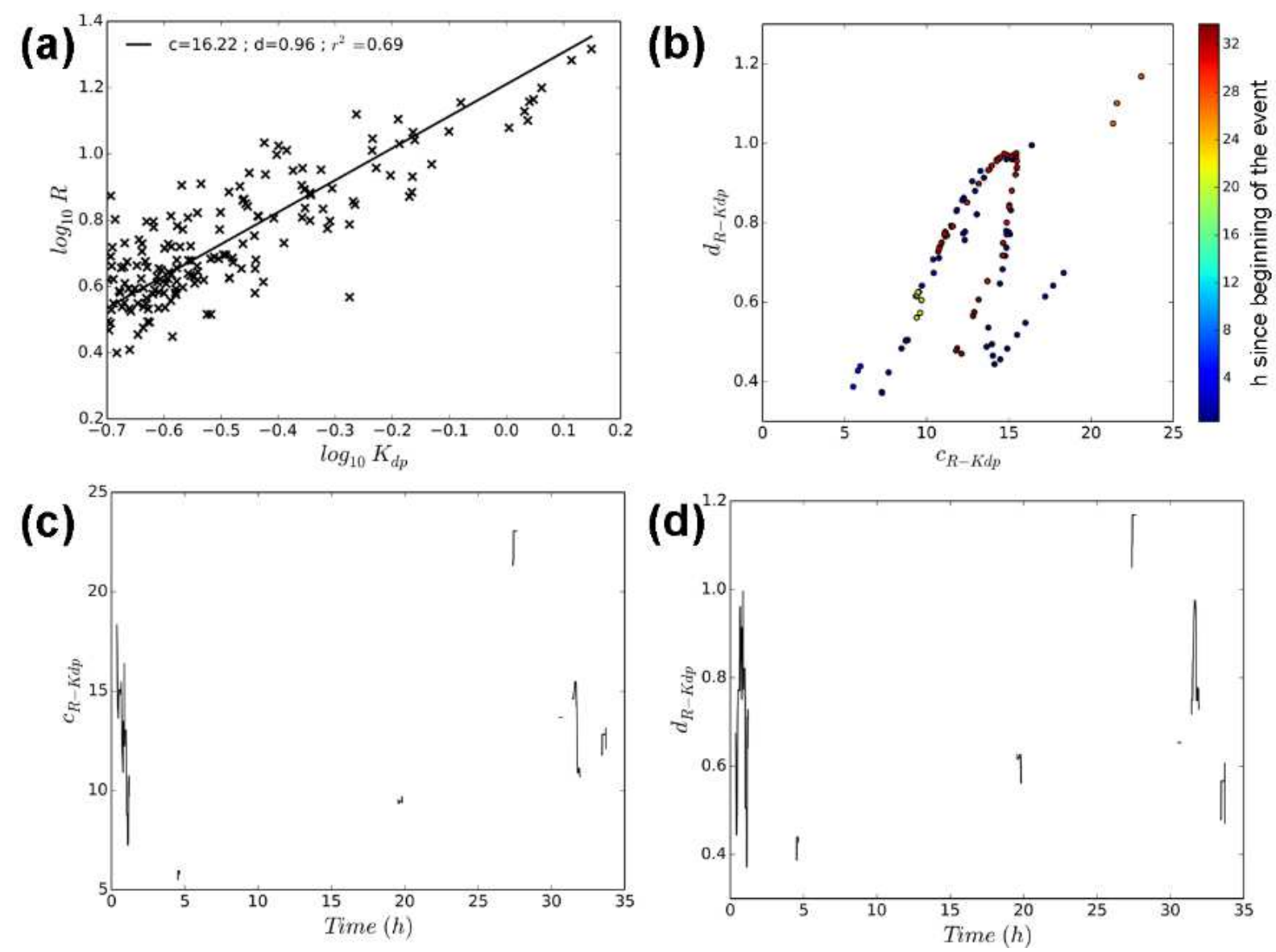

Figure 10: $R-K_{d p}$ relation for the 29-31 May 2016 event with Pars\#2. (a) Computation of the parameters $c$ and $d$ at the event scale (eq. 11 in a log-log plot). (b) Scatter plot of $d$ vs. $c$ estimated for each time step (20 minutes moving window). The colour of the points corresponds to the time within the event at which the parameters are estimated (c) Temporal evolution of $c$. (d) Temporal evolution of $d$. 

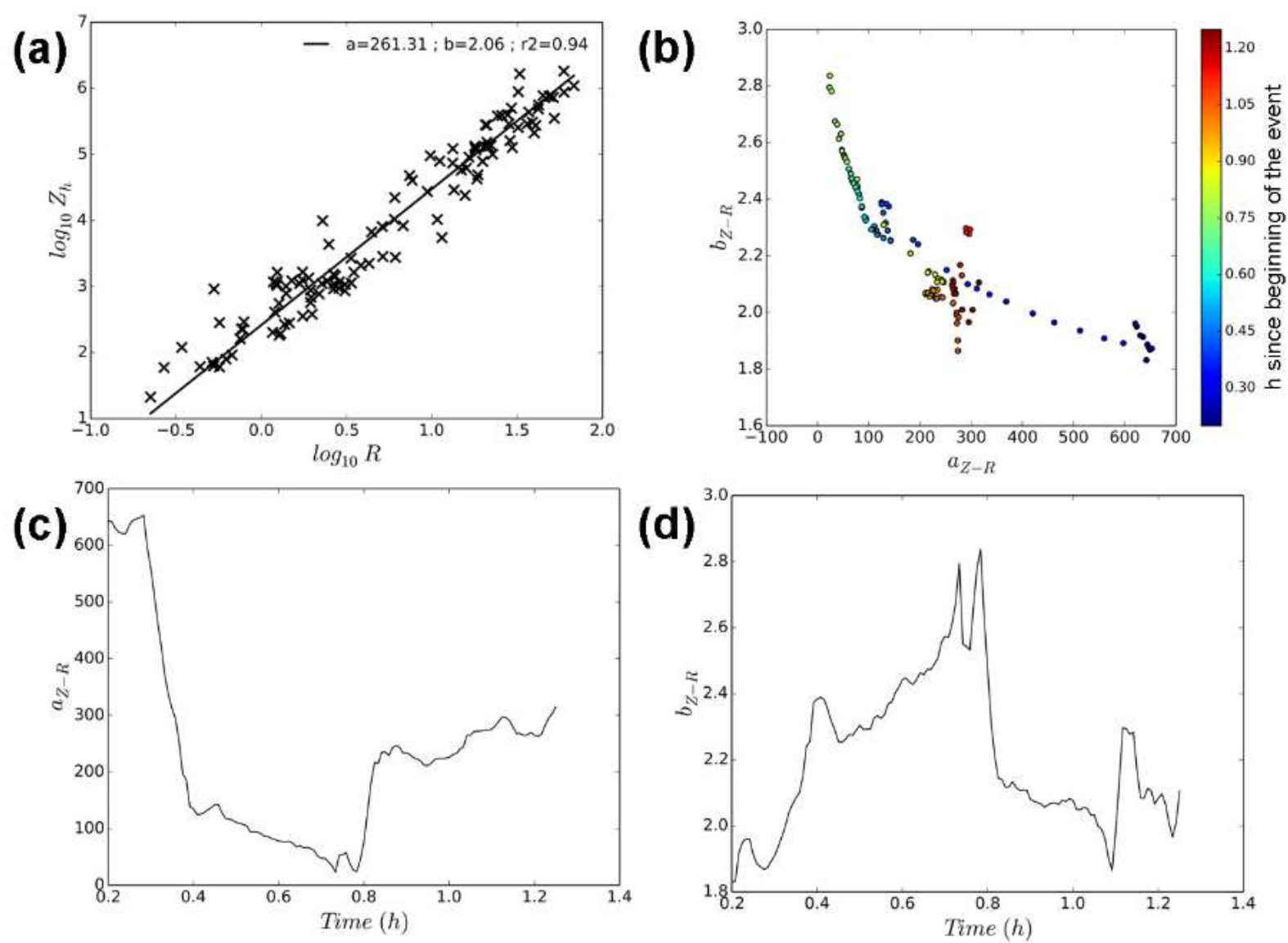

Figure 11: $Z_{h}-R$ relation for the 17 June 2016 event with Pars\#2. (a) Computation of the parameters $a$ and $b$ at the event scale (eq. 10 in a log-log plot). (b) Scatter plot of $b$ vs. $a$ estimated for each time step (20 minutes moving window). The colour of the points corresponds to the time within the event at which the parameters are estimated (c) Temporal evolution of $a$. (d) Temporal evolution of $b$. 

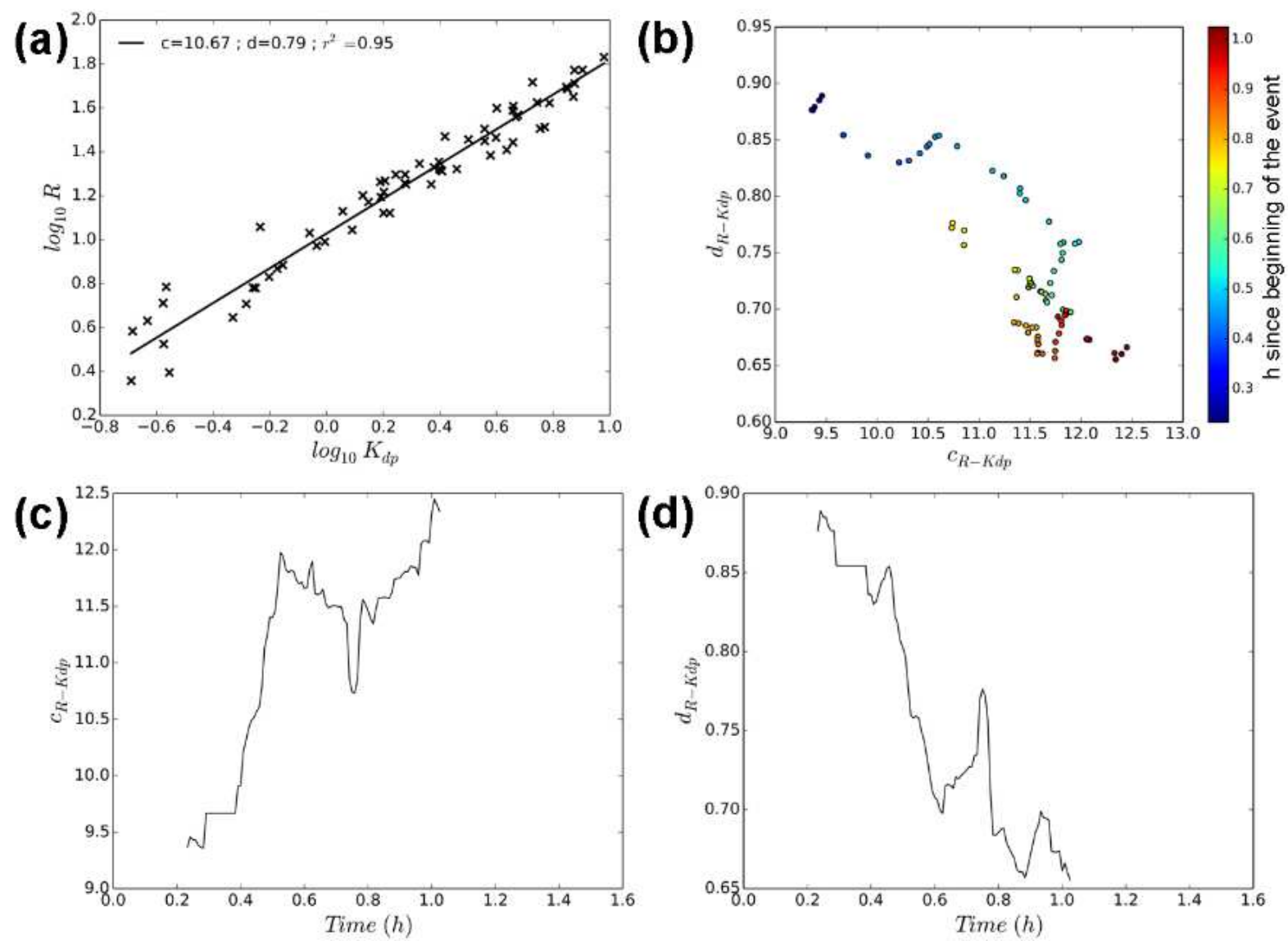

Figure 12: $R-K_{d p}$ relation for the 17 June 2016 event with Pars\#2. (a) Computation of the parameters $c$ and $d$ at the event scale (eq. 11 in a log-log plot). (b) Scatter plot of $d$ vs. $c$ estimated for each time step (20 minutes moving window). The colour of the points corresponds to the time within the event at which the parameters are estimated (c) Temporal evolution of $c$. (d) Temporal evolution of $d$. 

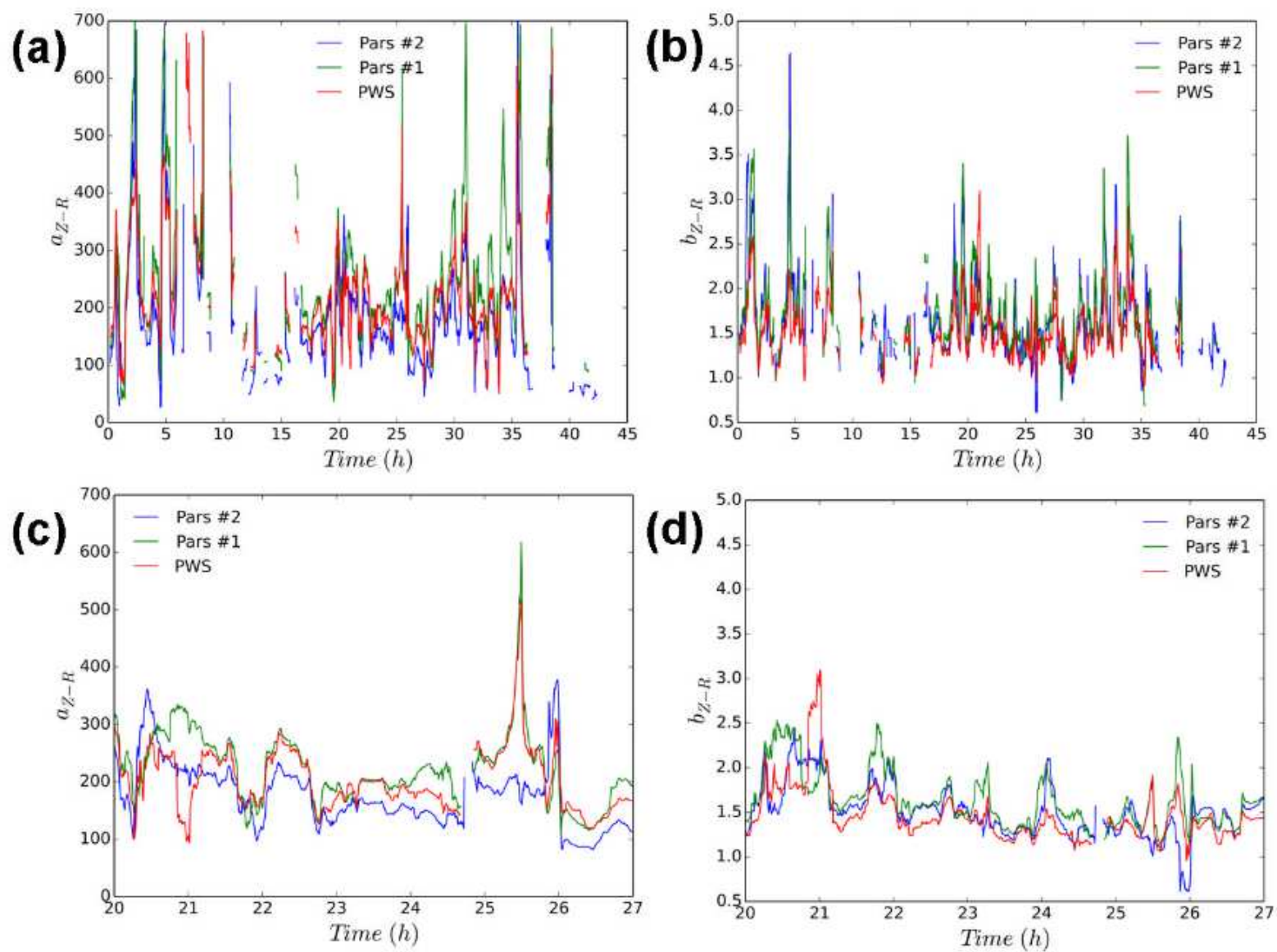

Figure 13: $Z_{h}-R$ relation for the 29-31 May 2016 event with the three disdrometers. (a) Temporal evolution of $a$ during the full event. (b) Temporal evolution of $b$ during the full event. (c) and (d) Zoom of (a) and (b) during a shorter period. 


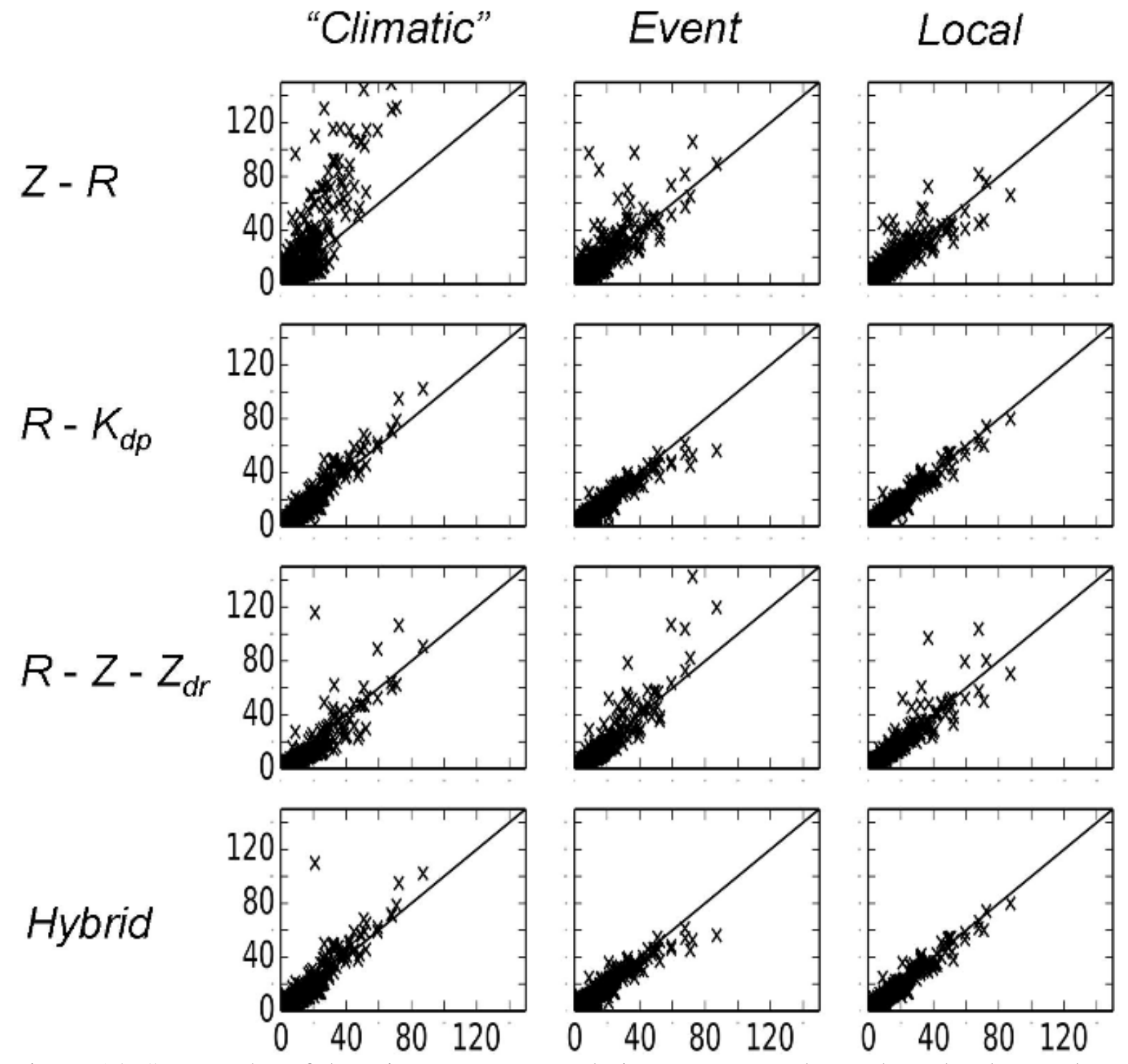

Figure 14: Scatter plot of the rain rates computed via reconstructed pseudo-radar data and algorithms $\left(R_{\text {rad }}\right)$ vs. the one obtained from direct drop measurements $(R)$ for the data collected with the help of Pars\#2 device. 

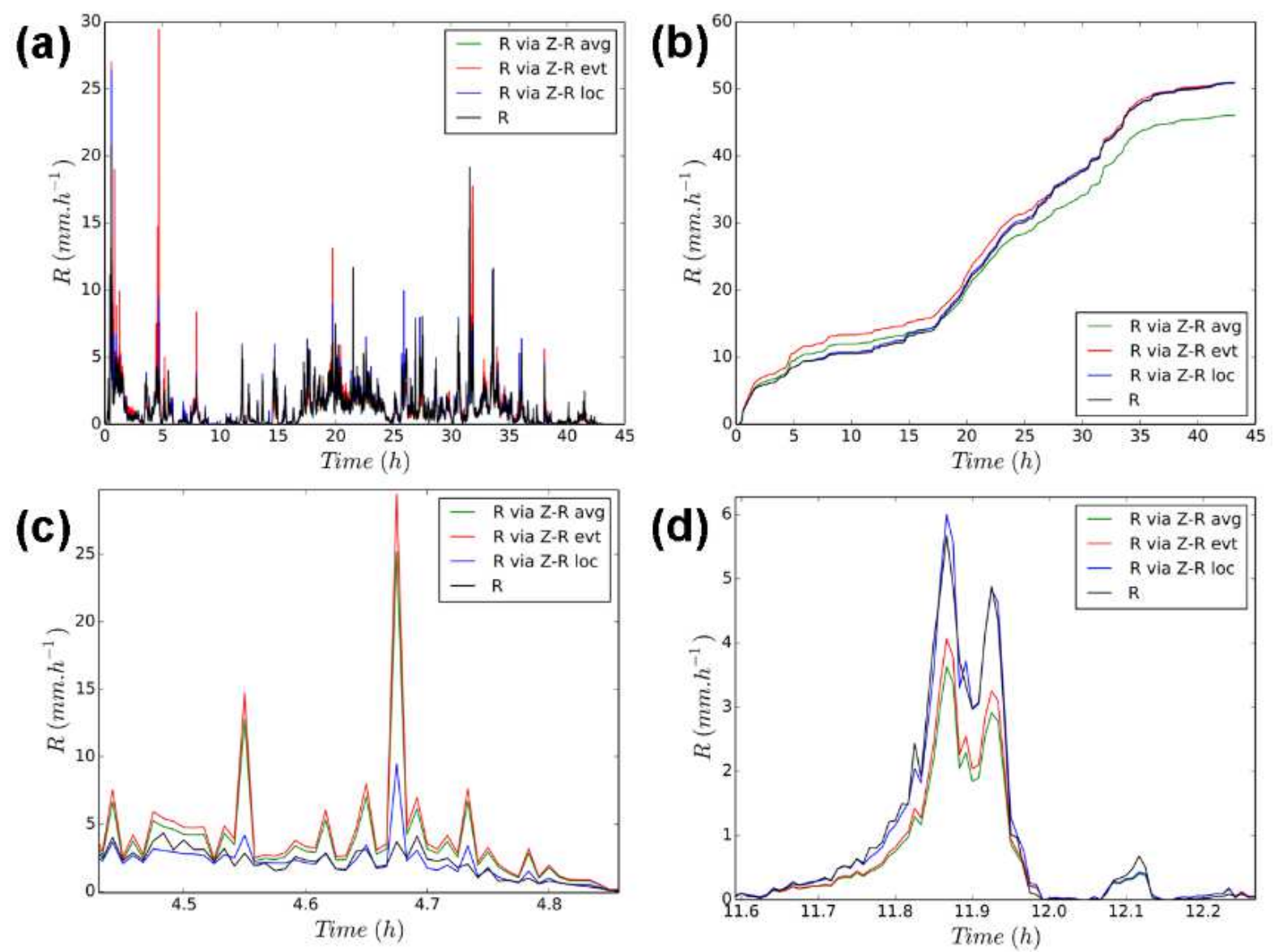

Figure 15: For the 29-31 May 2016 event and Pars\#2 data. (a) Temporal evolution of the rain rates obtained via reconstructed pseudo-radar data and the three $Z_{h}-R$ algorithms ("climatic", "event", and "local") along with the direct estimation of $R$. (b) Corresponding cumulative depth. (c) Zoom of (a) for a shorter period of time. (d) Zoom of (a) for another shorter period of time. 

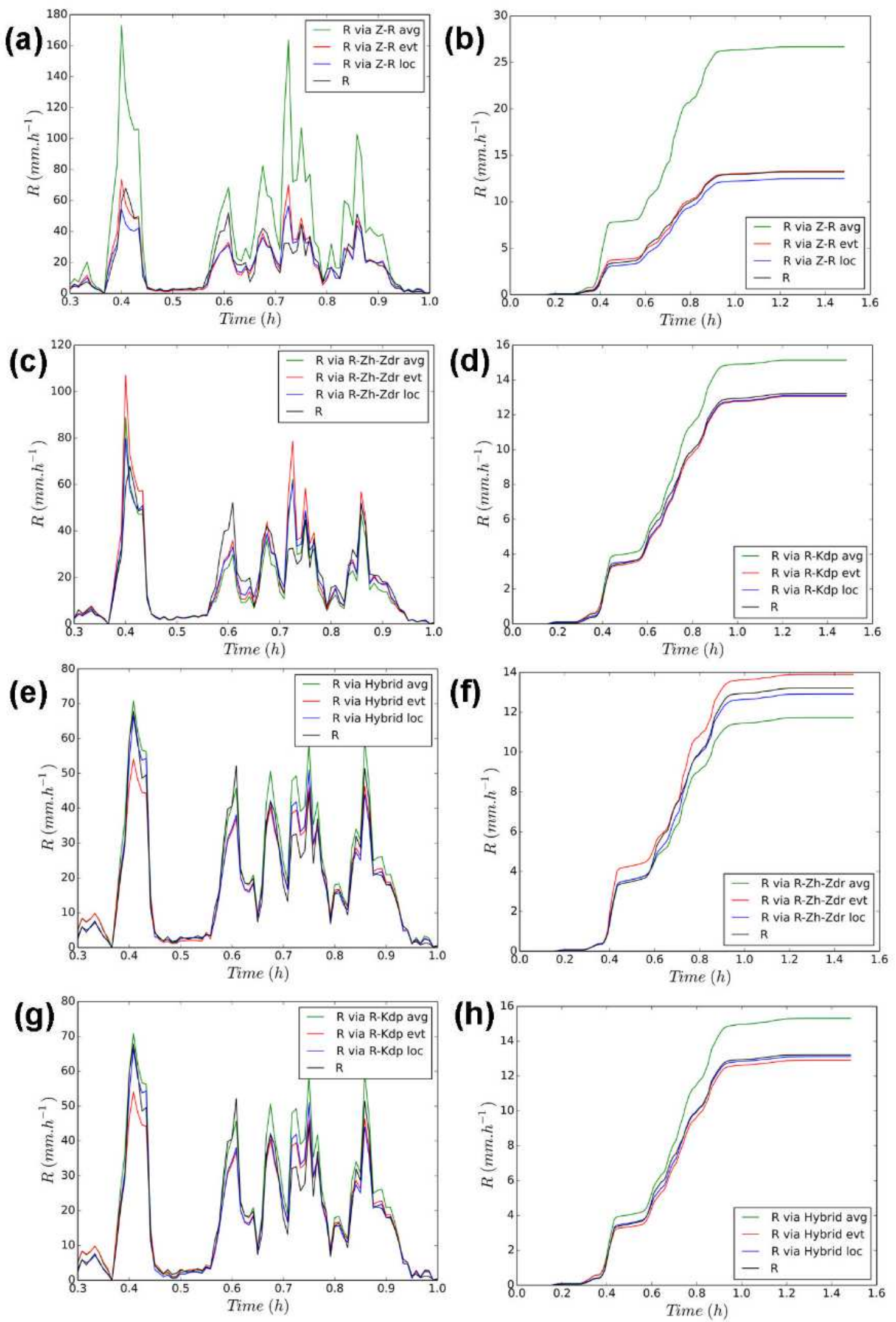

Figure 16: For the 17 June 2016 event and Pars\#2 data. (a) Temporal evolution of the rain rates obtained via reconstructed pseudo-radar data and the three hybrid algorithms ("climatic", "event", and "local") along with the direct estimation of $R$. (b) Corresponding cumulative depth. 